\title{
Resilience Modeling of Surface Transportation System in Mixed Traffic Environment
}

\author{
Shofiq Ahmed \\ sa0123@mix.wvu.edu
}

Follow this and additional works at: https://researchrepository.wvu.edu/etd

Part of the Civil Engineering Commons, and the Transportation Engineering Commons

\section{Recommended Citation}

Ahmed, Shofiq, "Resilience Modeling of Surface Transportation System in Mixed Traffic Environment" (2018). Graduate Theses, Dissertations, and Problem Reports. 3695.

https://researchrepository.wvu.edu/etd/3695

This Thesis is protected by copyright and/or related rights. It has been brought to you by the The Research Repository @ WVU with permission from the rights-holder(s). You are free to use this Thesis in any way that is permitted by the copyright and related rights legislation that applies to your use. For other uses you must obtain permission from the rights-holder(s) directly, unless additional rights are indicated by a Creative Commons license in the record and/ or on the work itself. This Thesis has been accepted for inclusion in WVU Graduate Theses, Dissertations, and Problem Reports collection by an authorized administrator of The Research Repository @ WVU. For more information, please contact researchrepository@mail.wvu.edu. 


\title{
Resilience Modeling of Surface Transportation System in Mixed Traffic Environment
}

\author{
Shofiq Ahmed \\ Thesis submitted to the \\ Benjamin M. Statler College of Engineering and Mineral Resources at \\ West Virginia University \\ in partial fulfillment of the requirements for the degree of \\ Master of Science \\ in \\ Civil Engineering
}

Kakan Dey, Ph.D., Chair

David R. Martinelli, Ph.D.

Dimitra Pyrialakou, Ph.D.

Department of Civil and Environmental Engineering

Morgantown, West Virginia
2018

Keywords:

Resilience, Connected and Automated Vehicle, Recovery budget, Recovery time, Mixedtraffic, Disaster

Copyright 2018, Shofiq Ahmed 


\section{ABSTRACT \\ Resilience Modeling of Surface Transportation System in Mixed Traffic Environment Shofiq Ahmed}

Large-scale natural disasters challenge the resilience of surface transportation system. The objective of this research was to develop a resilience model of surface transportation system in mixed-traffic environment considering varying Connected and Automated Vehicle (CAV) penetration scenarios. As deployment of CAVs are expected to improve traffic operations, a resilience model was developed in this research to evaluate the resilience performance of a transportation system with several CAV penetration levels $(0 \%, 25 \%, 50 \%, 75 \%$ and 100\%) for a given budget and recovery time. The proposed resilience quantification model was applied on a roadway network considering several disaster scenarios. The network capacity in terms of trips at any phase of disaster was compared to the pre-disaster trips to determine the system resilience. The capacity variation and the travel time variation was also estimated. The analysis showed that the resilience phenomenon of the transportation system improved with CAVs in respect of travel time and capacity improvement. The rate of improvement in link travel time for varied CAV penetration was almost identical for different disaster scenarios. For each disaster scenario, the individual link travel time reduced significantly with increased CAV penetration. However, higher penetration of CAVs (i.e., $50 \%$ or more), increased the recovery budget requirement. For example, the recovery budget needed for medium and large-scale disasters were $50 \%$ and $90 \%$ higher respectively compared to the recovery budget needed for a small-scale disaster. These higher costs were primarily needed for repair and replacement of intelligent infrastructure required for CAV. 


\section{ACKNOWLEDGEMENTS}

I would like to express my sincere gratitude to my supervisor, Dr. Kakan Dey, for his continuous and valuable guidance, counselling and inspiration throughout my graduate research work. Without his active interest, it would have been difficult for me to complete my research. I would like to thank Dr. David R. Martinelli and Dr. Dimitra Pyrialakou for being my thesis committee members and providing me with their utmost advice, support and encouragement.

Lastly, I would like to dedicate my thesis to my parents for instilling in me the importance of education, and to my siblings and wife for their limitless support. 


\section{Table of Contents}

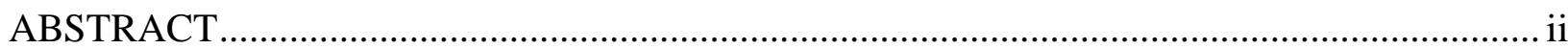

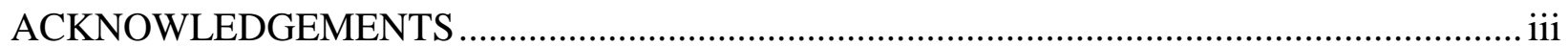

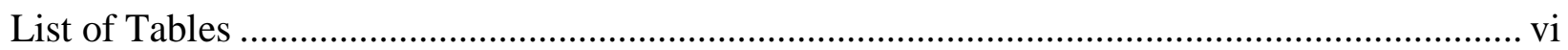

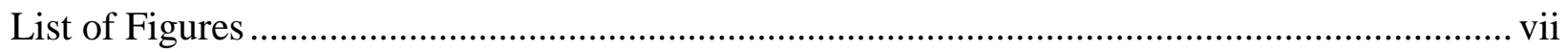

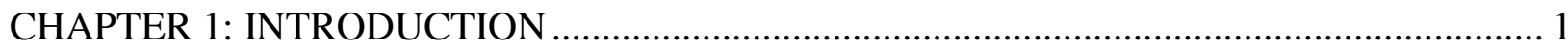

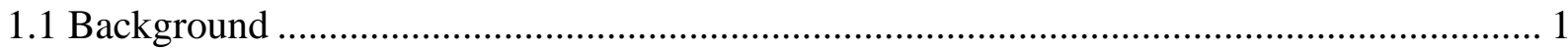

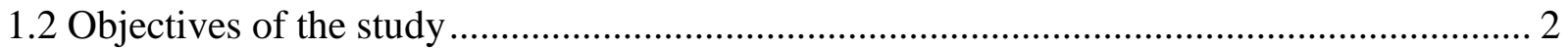

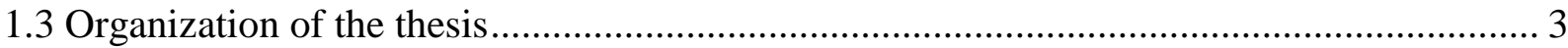

CHAPTER 2: LITERATURE REVIEW ..................................................................... 4

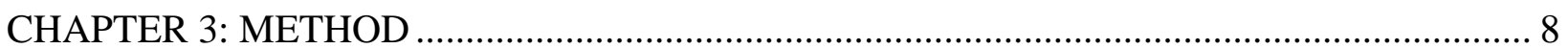

3.1 Preparation Phase (Phase 1, P1) .............................................................................. 9

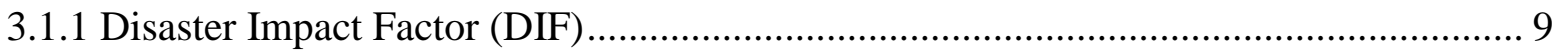

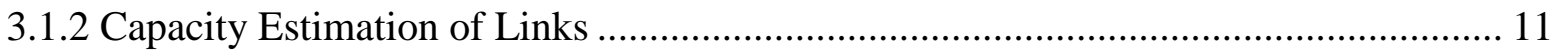

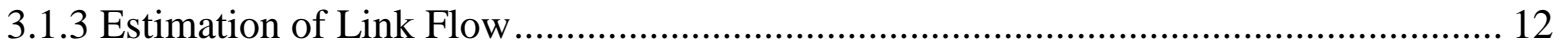

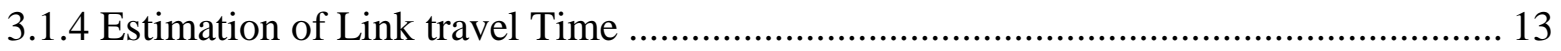

3.1.5 Estimation of Resilience Parameter.................................................................... 13

3.2 Immediate Before Disaster Impact Phase (Phase 2, P2) ............................................... 14

3.3 Disaster Impact Phase (Phase 3, P3) ................................................................ 14

3.4 Immediate After Disaster Impact Phase (Phase 4, P4)............................................ 15

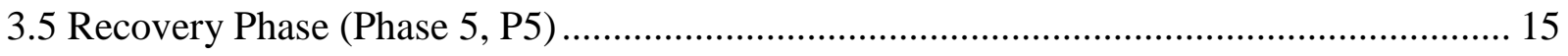

CHAPTER 4: DISASTER SCENARIO AND NETWORK SYSTEM.................................... 17

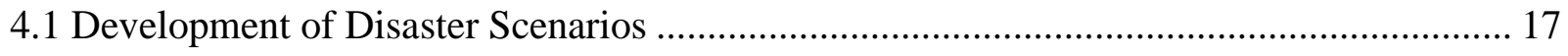

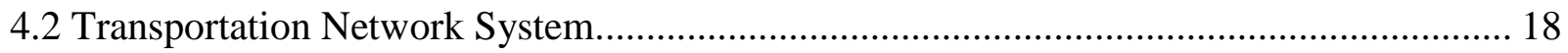


5.1 Disruption Impact Factor (DIF) Variation for Nodes ..................................................... 23

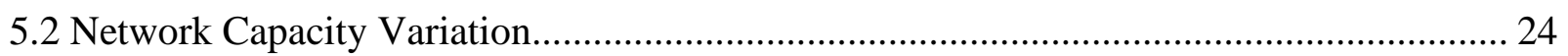

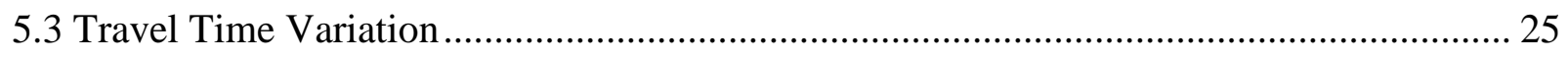

5.4 System Resilience (SR) Analysis ................................................................................ 31

5.5 Analysis of Recovery Budget and Recovery Time …………………………................. 32

CHAPTER 6: CONCLUSIONS AND FUTURE RESEARCH DIRECTIONS ........................... 34

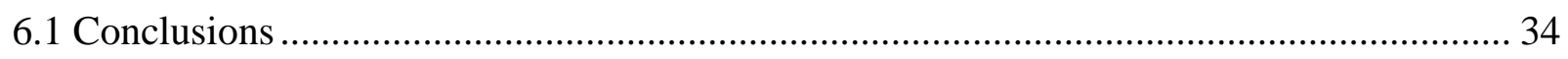

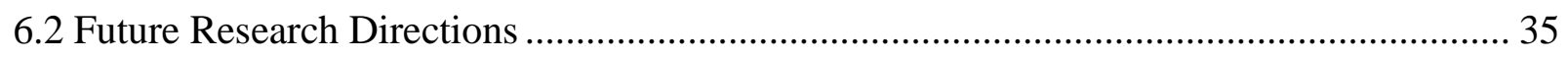

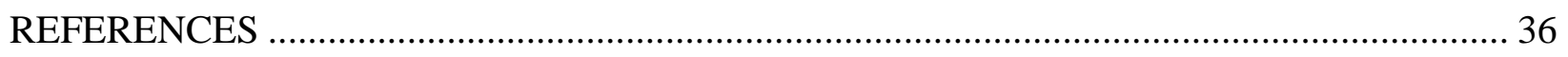




\section{List of Tables}

Table 4.1: Given performance parameters for Nguyen Dupuis network for pre-disaster condition for manual vehicles (adopted from [47]) ............................................................................. 20

Table 4.2: Origin-Destination (OD) distribution used in Nguyen Dupuis network ..................... 22 


\section{List of Figures}

Figure 4.1: Nguyen-Dupuis Network (adopted from [47]) ................................................. 18

Figure 4.2: Damaged nodes and links considered in P4 resilience analysis small-scale disaster scenario

Figure 4.3: Damaged nodes and links considered in P4 resilience analysis in medium-scale disaster scenario 19

Figure 4.4: Damaged nodes and links considered in P4 resilience analysis in large-scale disaster scenario 20

Figure 5.1: DIF ranking of nodes for different $\alpha, \beta$, and $\gamma$ combinations and average DIF ranking 24

Figure 5.2: Average capacity drop in mixed-traffic environment for varied disaster scenarios... 25 Figure 5.3: Individual link's travel time variation for $0 \% \mathrm{CAV}$ penetration due to the impact of the small-scale disaster scenario 26 Figure 5.4: Individual link's travel time variation for $25 \%$ CAV penetration due to the impact of the small-scale disaster scenario..... 27

Figure 5.5: Individual link's travel time variation for 50\% CAV penetration due to the impact of the small-scale disaster scenario 27 Figure 5.6: Individual link's travel time variation for $75 \% \mathrm{CAV}$ penetration due to the impact of the small-scale disaster scenario ..... 28 Figure 5.7: Individual link's travel time variation for $100 \%$ CAV penetration due to the impact of the small-scale disaster scenario 28 Figure 5.8: Individual link's travel time variation for medium-scale disaster scenario for 25\% CAV penetration.

Figure 5.9: Individual link's travel time variation for large-scale disaster scenario for $25 \% \mathrm{CAV}$ penetration. 29

Figure 5.10: Percentage increase in link travel time in P4 phase in small-scale disaster scenario with different CAV penetration 30

Figure 5. 11: Transportation system resilience variation for different disaster scenarios 31 


\section{CHAPTER 1: INTRODUCTION}

\subsection{Background}

The transportation system is designed to operate under normal weather conditions with limited consideration of extreme weather hazards. Thus, severe weather events such as large snowfall, heavy rainfall, hurricane, tornado, or even manmade disasters may cause significant degradation of the system performance. As the transportation system is one of the largest critical infrastructure systems, disruption may cause significant economic, social and financial impacts to the affected region. For example, in 2017, major 16 natural disaster events caused approximately $\$ 312.7$ billion worth of damage collectively to the transportation and infrastructure systems in the United States, and caused 362 fatalities [1]. Due to higher frequency of natural disasters in recent years, the consequences of disasters on transportation systems has received increasing attention from policy makers and researchers. Transportation system's performance is critical to disaster response such as evacuation operation before disasters, rescue operations, and recovery activities during and after disasters. Resilience of a transportation system defines the inherent quality of the system to recover from unwanted consequences. To address this need, researchers have formulated resilience quantification models and strategical mitigation approaches to estimate the resilience of a transportation system to improve system resilience and reduce impact and duration of disaster recovery time (for a comprehensive discussion see [2]). Though impact of different types of disaster events were considered in past studies to estimate the transportation system resiliency, the impacts of intelligent transportation system (ITS) were investigated in very limited scale. As sensors, computing platforms and communication networks play an important role in ITS functionalities of modern ground transportation system [3], cybersecurity and failure risk of backbone infrastructure has become a critical issue $[4,5,6]$. The security implications of compromising a car's internal communication network can lead to crash [5]. Furthermore, as observed in the past, hacking of the roadside signs or ITS components such as variable message signs (VMS) can lead to unwanted consequences (e.g., congestion or inappropriate message/misinformation display) [4]. Similarly, security of future vehicle-to-vehicle (V2V) and vehicle-to-infrastructure (V2I) communication systems of CAV can be common cyber security target and must be secured to eliminate all potential cyber-attacks [5, 7]. However, recent trends 
in the impact of flooding caused by the severe hurricane or other natural disaster scenarios has been the prime concern of this research work. Therefore, this work has considered the impact of natural disaster scenario such as hurricane, flooding etc. in their model development in which roads and infrastructural elements (i.e. road side unit) become dysfunctional or damaged.

Deployment of ITS features (such as connected and automated vehicles, intelligent signal systems, and adaptive safety solution) in traditional transportation systems can alter the resilience phenomenon and system performance metrics. In recent years, significant advances were made in connected and automated vehicle (CAV) technologies, with expected deployment within the next decade. It is important for transportation professionals to study these new technologies to understand both potential benefits and negative consequences. In general, CAVs rely on their V2V and V2I communication, and a diverse array of sensors for driverless navigation on roadways. The first generation of CAVs will interact with other CAVs and non-CAVs, herein considered a mixedtraffic environment. As CAV operational efficiency depends on ITS infrastructure, such as road side V2I communication infrastructure, transportation agencies need to install and maintain these devices to support CAVs. Researchers have quantified the resilience of the conventional transportation system (i.e., system with non-CAV only) in many literatures (for example, [8, 9]). Further, several recent studies have analyzed the performance of CAV operation in a natural disaster scenario, and in a mixed-traffic environment (e.g., $[10,11])$. However, no study investigated the implication of CAV in quantifying transportation system resilience considering travel time and capacity implications and network resilience performance. This study developed a resilience estimation framework that can be used to analyze resilience of surface transportation system in a mixed-traffic environment considering different disaster scenarios and CAV penetration levels. In general, the natural disaster impacts on the transportation system include the closure of the lane(s) or roadway segments due to flooded conditions, pavement damage, broken or dysfunctional signal systems and other CAV road side units, and lead to capacity and flow reduction.

\subsection{Objectives of the study}

The primary objective of this study was to develop a new resilience model to analyze the transportation system, considering a mixed-traffic environment including both CAVs and non- 
CAVs to assess and quantify the transportation system's performance metrics and resilience. The model was formulated as a combination of transportation system performance matrices. Along with the resilience characteristics, the critical role of recovery budget and recovery time on postdisaster transportation system performance was also evaluated in a mixed-traffic environment.

The specific objectives of the study were as follows:

- Investigate the criticality of network nodes due to the variation of the disaster impact,

- Investigate the variation in link travel time for different penetration of CAV environment due to the influence of the different disaster scenarios,

- Estimate the resilience of the transportation network system due to different disaster impact scenarios, and

- Evaluate the impact of recovery budget and time on post disaster transportation system performance.

\subsection{Organization of the thesis}

The thesis is composed of six chapters. Chapter 1 provides a brief introduction on importance of transportation system resilience and motivation of the research and presents the research objectives. Chapter 2 reviews past research on resilience in the transportation domain and the impacts of ITS and CAV technologies on the transportation system's resilience. Chapter 3 describes the components of the proposed resilience model and the mathematical formulation of transportation system resilience in a mixed-traffic environment (i.e., CAV and non-CAV). Chapter 4 presents the hypothetical disaster scenarios and the studied transportation network system. Chapter 5 describes the application of the proposed resilience models on the hypothetical transportation network and reports the results. Chapter 6 summarizes the conclusions and identifies future research directions. 


\section{CHAPTER 2: LITERATURE REVIEW}

Implementation of connected and automated vehicle (CAV) in the transportation system will affect the resilience performance metrics of the transportation network. Based on the literatures, CAV deployment, in general, will increase the capacity of signalized intersections $[12,13]$, improve weather condition data collection and prediction [14], change driving behavior such as reduced headway [15, 16], improve adaptive cruise control performance [17, 18], improve advanced traveler information systems [19, 20], and improve traffic management system performance [21].

Many studies on resilience relied on the stepwise activity-based resilience model development considering network travel time, demand and capacity in the context of disasters [8, 22]. For example, the Networked Infrastructure Resiliency Assessment (NIRA) framework can be used to analyze resiliency of any network based infrastructure system such as a transportation system, energy system, or communication system [22]. Resilience was quantified and formulated in the work as an optimization problem to minimize the average travel time between the origin and destination [22]. Faturechi and Miller-Hooks formulated a nonlinear, stochastic and bi-level model for Travel Time Resilience Problem (TTRP). Origin-Destination (O-D) trips travel time were considered as the resilience parameter. Within this bi-level model, the expected value of network system resilience was maximized in the upper level for all given scenarios, and the flow pattern was optimized in the lower level to determine the link flows considering a partial user equilibrium $[8]$.

Other studies proposed an optimization-based resilience model to quantify transportation system resilience [9, 23, 24]. Considering budget, time, and resource constraints, Chen and MillerHooks formulated a stochastic mathematical program to quantify resilience level through maximization of travel demand that could be satisfied in post disaster scenario [9]. Although [8] discussed resilience in a simple hypothetical road network system, [9] considered an inter-modal freight system of the western United States (US) in their model performance evaluation. The resilience model analyzed several natural and man-made disaster scenarios (e.g., bombing, terrorist attack, flood, earthquake) and showed that the resilience level could be improved by 57\%, on average, due to the level of recovery effort [9]. As an extension of this study, Miller-Hooks et al. formulated the resilience parameter as the maximum post-disaster flow of shipments in the network for disruption scenario and developed the resilience model to measure and maximize the 
resilience of freight transportation networks [23]. In addition to travel time as the key resilience measure, Donovan and Work considered travel time per mile (i.e., pace) variation to assess the resilience of transportation network [24]. This study used GPS datasets from taxi trips in the New York City, NY and showed that Hurricane Sandy and Hurricane Irene increased per mile travel time by two minutes and 40 seconds respectively [24].

Beyond estimating resilience of a system, some studies evaluated the impact of the investment decisions on infrastructure and proposed resilient investment model to improve the performance of networked infrastructure from disruptions. For example, [25, 26] proposed a system performance evaluation model considering the impact of investment decisions to protect and improve the transportation infrastructure performance from future natural disaster events. The impacts of probable flood scenarios based on the prediction of sea level rise (SLR) datasets on Washington, DC transportation system were considered. [26] showed the impacts of protective infrastructure (e.g., flood barrier/dam, elevating streets or rails, increasing pumping capacity of tunnels) in reducing recovery cost. It was estimated that by implementing chosen protective infrastructure investment for forecasted future climatic events, recovery cost can be reduced by 54\% [26]. Asadabadi and Miller-Hooks studied the maritime transport resilience and optimized a cooperative and competitive investment program among ports within a maritime transportation network to improve the port throughput performance in disaster scenarios [27].

Several post-disaster survey-based studies designed real world implications of different pre and post disaster activities that might be crucial in disaster scenarios to measure the transportation system performance. For instance, Kontou et al. conducted a survey to identify commuters' trip making behavior to measure the transportation performance disruption after Hurricane Sandy [28]. According to [28], parents with highr number of children got back to normal working schedule where household with higher income relied on telecommuting longer in post-disaster situation. Freckleton et al. proposed a resilience model to estimate transportation network performance [29]. Sixteen variables were used including transportation system parameter (such as level of service, travel time, or mode of transport) and disaster response (such as resource availability, emergency response) to determine the ultimate network resiliency. Each parameter was assigned a quantitative or qualitative measure and based on the combination, the resilience of the network was estimated as low, moderate, high or very high [29]. 
Other than road-based network system, several studies evaluated the resilience of air transportation system. For example, [30, 31] proposed an airline flow-based resilience model considering the number of arrival and departure flows on an air-hub (airport) to quantify the air transportation network system resilience. While Janić measured resilience for an air transport network (consisting of multiple airports) [30], Faturechi et al. presented resilience model to measure resilience for the pavement network of an airport [31].

As CAV deployment is expected to impact the transportation system performance, several studies have considered a mixed-traffic environment with different penetration rates of CAVs, and estimated the level of service (LOS) [10, 32, 33]. Several studies investigated the impacts of CAVs during natural disasters such as flood or earthquake $[11,34]$. Zhu and Ukkusuri proposed a car following model parameter estimation model in a no-disaster mixed-traffic (i.e., connected and non-connected vehicles) environment and measured the travel time and travelled distance using connected vehicle data [10]. Another study proposed a framework using connected vehicle (CV) technology to optimally route the vehicles in a flooding scenario applying the time-dependent hyper-star algorithm [11]. Researchers also showed that a higher penetration of CVs and longer evacuation time improves the evacuation operation in a CV environment [34].

Few research articles and reports were also explored to review the infrastructure requirement of CAVs and to understand better the implications of CAV in transportation system performance during disaster scenarios. For instance, [35] discussed, in general, policy related facts (i.e. road signage and markings requirement) for the implementation of CAV. [36] developed the optimal strategy to allocate the emergency resource including fire safety team and equipment, and ambulances to maximize the service oriented coverage to the critical transportation infrastructures. Further, [37, 38] designed the impacts of pre-positioning of emergency supplies and protective resources to reduce the disaster consequences.

Time step conceptual failure analysis model [39] was evaluated in this study to generate the relative impact of failed components within the network system. [40] evaluated the lane merging implication for mixed-traffic scenario and provided a general headway scenario for traffic stream which was adopted in this study to calculate the average headway of mixed-traffic. [41, 42] discussed the factors that can influence the capacity of a road link most and recommended that number of lanes and lane widths were the most influential factors. For a comprehensive review on 
resilience parameters, [44] was reviewed and to develop comprehensive disaster scenarios, this study relied on few national research reports on the real disaster impact (for example, [45, 46]). Finally, to analyze the model implication on a case study or network system, this study considered the Nguyen-Dupuis network system and it's characteristics from [47]. [47] modeled the optimal plate scanning location for various origin-destination demand scenarios.

Though there were numerous research publications on resilience quantification of surface transportation system considering conventional traffic (i.e., non-CAV), no study attempted to study the resilience of a transportation system holistically in a mixed-traffic environment. A new resilience model was proposed to quantify the transportation system resilience performance metrics in the mixed-traffic environment in this study. 


\section{CHAPTER 3: METHOD}

Different sensors, V2V and V2I communication system of CAVs will alter key traffic parameters and patterns that are related to the performance of a transportation network. Key parameters and patterns include travel demand (e.g., population with disability can make trips with CAV), level of service (LOS) improvement due to increased roadway capacity, condition of road links, and travel time reduction due to real time shortest route selection. In general, due to the disaster impact: (i) some trips can get cancelled due to the failed nodes/links along the route; (ii) some trips cannot occur due to unavailability of routes; and (iii) many trips will take longer travel time due to the disaster impact.

While several researches considered travel time parameter to estimate transportation system resilience through measuring travel time changes due to the disaster [8, 24], others expressed resilience as the trip handling capacity of the network in post-disaster scenario compared to pre-disaster scenario $[9,23,30,31]$. As road link capacity improvement, travel time reduction, efficient and safe driving are expected to be key improvements CAV brings to the transportation system, in this study, the transportation system resilience was estimated based on the traffic flow variation, travel time variation, and capacity variation in a mixed-traffic environment. The proposed resilience model considered these potential improvements in the evaluation of transportation system resilience. Furthermore, CAV systems are expected to rely on detailed mapping of the road network including infrastructural components such as road side units (RSUs), advanced signal operation system, digital road markings and signage [35]. The rapid repairs of affected transportation network in the disaster recovery stage is critical to support CAVs operations. To account for these repairs, a Disruption Impact Factor (DIF) was introduced in this work. This factor expressed the criticality of nodes (i.e., intersections) in terms of disaster impact and can be used to prioritize most critical nodes for repair within the given budget. Several past

research articles divided disaster durations in three stages [8, 31]. However, in these three-stage classification, there is no defined stage which investigates the system's network condition during disaster landfall and after the recovery activities. To consider these two distinct disaster phases, the disaster timeline is divided into five disaster phases in this model to analyze the performance of transportation system in smaller timescales. These five phases included a preparation phase, immediately before disaster impact phase, a disaster impact phase, immediately after disaster 
impact phase, and a recovery phase. The formulation of performance metrics and the detailed explanation of the five phases are discussed in the following subsections.

\subsection{Preparation Phase (Phase 1, P1)}

Numerous works studied network fortification by pre-planned resource allocation and reinforcing network components in the disaster preparation phase [for example, 23, 36, 37]. In this work, preparation phase does not necessarily mean that there is a forecasted disaster or that preparedness activities need to be undertaken. Rather, this phase represents the importance of understanding the pre-disaster normal traffic condition of the transportation network system and its performance level in terms of resilience parameter so that if any disaster happens the impact of disaster on system performance can be estimated and quantified. Though the preparedness activity such as: development of evacuation plan and training teams for rapid recovery [31], development of

effective disaster management technique through pre-planned resource allocation [38], high resolution forecasting of the disaster to estimate the disaster impact [25, 26] are critical components in the preparation phase before any disaster happening, this study has not investigated the implication of evacuation strategy. Rather, only the estimation of the resilience parameters is considered in preparation phase in mixed traffic environment. In this section, the parameters that influence the performance of the system and will be impacted by the CAV presence in before, during, after disaster scenarios are expressed to represent these impacts. The formulation of the performance metrics is elaborately discussed in the following sub-sections.

\subsubsection{Disaster Impact Factor (DIF)}

Because the propagation of disruption or disaster in the transport network over time is a critical factor in resilience analysis (i.e., the relative temporal impact of the disaster in different nodes), the researchers defined a disruption impact factor (DIF) based on the time step conceptual failure analysis proposed in [39]. The time step technique through failure analysis in [39] showed that failure of a single node can trigger the failure of a system of nodes and the failure pattern will be propagated in several time steps based on the connection pattern between nodes. For example, consider that during time step $t_{0}$, node $\mathrm{a}_{0}$ is impacted. If $\mathrm{a}_{0}$ is connected to $\mathrm{a}_{1}$ and $\mathrm{a}_{2}$, then in time step $t_{1}$, nodes $a_{1}$ and $a_{2}$ could be impacted. Consequently, in time step $t_{2}$, the nodes connected to $a_{1}$ 
and $\mathrm{a}_{2}$ could be impacted. Considering this failure pattern, a DIF was calculated. However, the consideration that the nodes connected with the impacted node are going to be impacted at the same time is not reasonably realistic. The time duration to propagate the impact to other nodes could depend on several factors such as; traffic flow, average velocity, length of the link between the nodes. Equation 1 shows the formulation of DIF of node. DIF explains the geometric pattern of the transport network and the inherent quality of the network to survive the disruption (based on the connection pattern of nodes and which node has been affected first by the disruption). Any node can be impacted either directly by the disaster or due to the influence of the impacted neighbor nodes. DIF of a node was formulated considering the influence of the connected links of that node. The least time duration to get impacted for any node depends on the length of the neighborhood links, average link speed, and traffic flow on connected links. Therefore, DIF of a node was formulated as a combination of length, average speed, flow of the connected links by assigning relative weight to each parameter.

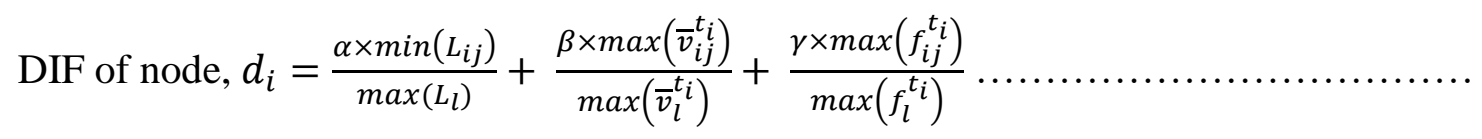

Where,

$j=$ Neighborhood nodes

$L_{i j}=$ Length of link from node $i$ to $j$

$L_{l}=$ Length of link $l$

$\bar{v}_{i j}^{t_{i}}=$ Average velocity on the link from node $i$ to $j$ during time $t_{i}$

$\bar{v}_{l}^{t_{i}}=$ Average velocity on link $l$ during time $t_{i}$

$f_{i j}^{t_{i}}=$ Flow on the link from node $i$ to $j$ during time $t_{i}$

$f_{l}^{t_{i}}=$ Flow on link $l$ during time $t_{i}$

$\alpha, \beta$, and $\gamma=$ the relative weights of the three parameters. Each parameter could range from 0 to 1 , where higher is more important and where, $\alpha+\beta+\gamma=1$. Based on the variation of relative weight, the DIF values for a node can change, which implies that the criticality of nodes in terms of the disaster impact can vary depending on the relative weight of the considered parameters. Deciding on which value should be used for $\alpha, \beta$, and $\gamma$ is critical. Different traffic agencies may 
take different initiative to prioritize among these three factors based on their geographical pattern of the road network, commuters' trip making behavior, and traffic demand. In general, traffic flow is considered to be the most important and influential parameter in evaluating the system performance.

\subsubsection{Capacity Estimation of Links}

Reduction of average headway in a mixed traffic compared to non-CAV condition increases the roadway capacity. To estimate the capacity improvement, with the varying penetration rate of $\mathrm{CAV}$, the saturation headway of mixed traffic was measured using the following equation 2 collected from [40],

$$
\text { Headway of mixed-traffic, } \bar{h}_{m i x}(P)=P^{2} \bar{h}_{A A}+P(1-P)\left(\bar{h}_{A M}+\bar{h}_{M A}\right)+(1-P)^{2} \bar{h}_{M M}
$$

Where,

$P=\mathrm{CAV}$ penetration rate

$\bar{h}_{A A}=$ Average headway for a CAV following a CAV

$\bar{h}_{A M}=$ Average headway for a CAV following a non-CAV vehicle

$\bar{h}_{M A}=$ Average headway for a non-CAV following a CAV vehicle

$\bar{h}_{M M}=$ Average headway for a non-CAV vehicle following a non-CAV vehicle

According to [40], CAV can follow three headway scenarios; neutral, conservative, and aggressive. The researchers in this study considered the gradual change in headway preference from conservative to aggressive depending on the CAV penetration rate following the recommendation of study [40]: 0-25\% CAV penetration will follow $\bar{h}_{A A}=0.45 \operatorname{secs}, \bar{h}_{A M}=\bar{h}_{M A}=$ $\bar{h}_{M M}=1.8 \mathrm{secs} ; 26-50 \% \mathrm{CAV}$ penetration will follow $\bar{h}_{A A}=0.40 \mathrm{secs}, \bar{h}_{A M}=1.6, \bar{h}_{M A}=\bar{h}_{M M}=1.8$ secs; $51-75 \% \mathrm{CAV}$ penetration will follow $\bar{h}_{A A}=0.35 \mathrm{secs}, \bar{h}_{A M}=1.4, \bar{h}_{M A}=\bar{h}_{M M}=1.8 \mathrm{secs}$; and 76-100\% CAV penetration will follow $\bar{h}_{A A}=0.30 \mathrm{secs}, \bar{h}_{A M}=1.2, \bar{h}_{M A}=\bar{h}_{M M}=1.8$ secs.

The capacity of link $l$ depends mostly on the number of lanes and lane width [41, 42]. According to [42], lane width has a linear relationship with capacity. As during any disaster scenario, the operation on any lane can be disrupted, number of functional lanes becomes critical 
in estimating capacity. The capacity of link $l$ in this work was measured as of the following equation 3, 4, and 5. Standard lane width was assumed to be $12 \mathrm{ft}$.

Capacity of link $l, C_{l}^{t_{i}}=N_{l n, l} \times \bar{C}_{l n, l}^{t i}=N_{l n, l} \times \delta_{l n} \times\left(1-\frac{6}{100} \times N_{\ln , l}\right) \times f_{l n-w} \times \bar{C}_{l n} \ldots$

Where,

Capacity adjustment factor for lane width, $f_{l n-w}=1-\frac{\text { actual lane width }}{\text { Standard lane width }} \ldots \ldots \ldots . . .$.

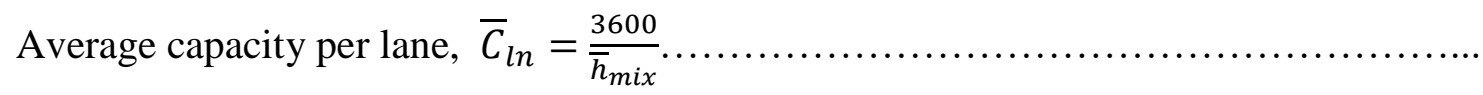

$\bar{C}_{l n, l}^{t i}=$ Average capacity per lane in link $l$ during time $t_{i}$

$N_{\text {ln,l }}=$ Number of lanes in link, $l$

$\delta_{l n}=\mathrm{A}$ binary factor to represent lane's functionality, if a lane is operational then $\delta_{l n}=1$, otherwise 0 .

\subsubsection{Estimation of Link Flow}

Traffic flow on a lane or link can be expressed by equation 6 and 7. Traffic flow shows significant temporal distribution in different scenario (i.e., disaster impact level) and traffic condition (e.g., congestion). For example, traditionally traffic flow is significantly less during midnight compared to morning peak and evening peak [43]. Furthermore, during the event of any forecasted disaster, the temporal variation of flow could be significantly different from the traditional situation. In this research, largest peak flow (between AM peak and PM peak) was considered as peak flow of lane $l n$ in link $l$ during time $t_{i}$ and flow in any link $l$ was expressed as the summation of the flow of lanes (equation 7). During or after disaster, traffic flow pattern of the network system can radically change due to the damage to lanes or in some instances, the whole link can be dysfunctional causing the deviation of routes for certain O-D pairs.

Flow of lane $l n$ in link $l$ during time $t_{i}, f_{l n, l}^{t_{i}}=\delta_{l n} \times p_{l n, l}^{t_{i}} \times f_{l n, l}^{\text {peak }} \ldots \ldots \ldots \ldots \ldots \ldots . . . . . . .$.

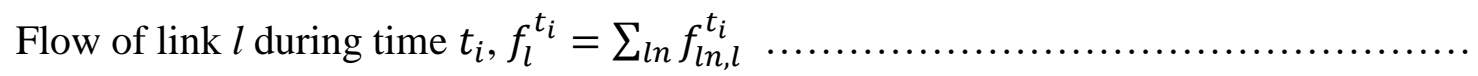


Where,

$f_{l n, l}^{\text {peak }}=$ Peak flow of lane $\ln$ in link $l$

$p_{l n, l}^{t_{i}}=$ hourly flow rate as a percentage of peak flow

\subsubsection{Estimation of Link travel Time}

Typically, every trip takes the shortest route between origin-destination (OD) in normal road weather condition. However, depending on different disaster scenario impacts and traffic condition (e.g., congestion), trip makers' route preference changes. Consequently, average travel time of a trip or link travel time changes with the route preference. Furthermore, due to the impact of the disaster in mixed-traffic situation, two situations may arise that includes the complete failure of some nodes and links (either structurally or functionally) which will impact some OD pairs' route preferences and trip decision. Capacity reduction of links will result in more congestion or reduced LOS. Both situations will attribute to increase in link travel time and total trip time. The updated travel time of trips and links was measured applying equations 8 and 9.

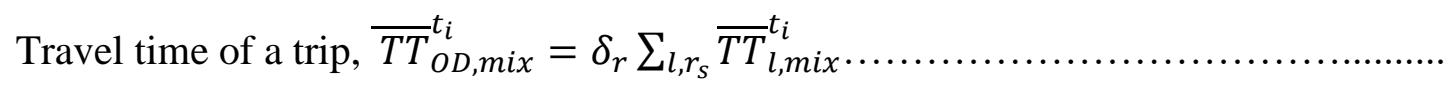

Where,

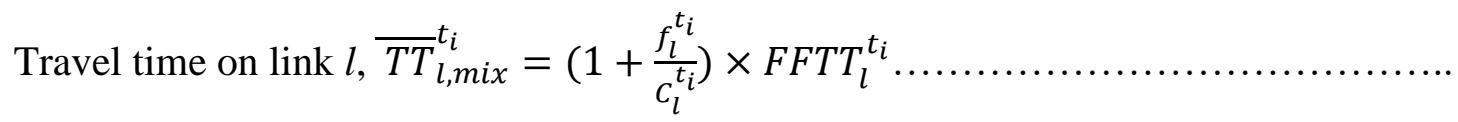

Here,

$\mathrm{FFTT}_{l}^{t_{i}}=$ Free flow travel time on link $l$ during time $t_{i}$

$\delta_{r}=$ A binary factor to represent availability of route, if a route is available for an origindestination combination, then $\delta_{r}=1$, otherwise 0 .

$r_{s}=$ Shortest route

\subsubsection{Estimation of Resilience Parameter}

As resilience is a system characteristic [44], the system resilience (SR) parameter was introduced and expressed in this research based on the disruption of the network's OD trip matrix. Since, trip handling capacity of a network is a good estimator of the network's performance, this study used 
the reduction in trip handling capacity to quantify system resilience. Hence, SR was considered as the ratio of the number of trips in any disaster phase that can be accommodated in that phase to the number of the pre-disaster OD trip matrix in the system and was expressed as of the following equation 10. Here pre-disaster trips were represented as the total number of trips in peak hour in a typical demand scenario for the network and expressed as the trip handling capacity of that network. As trip handling capacity of a network in pre-disaster scenario is the maximum number of trips that are served by the network, the SR in pre-disaster phase is equal to 1 and SR for any other phase can be defined as-

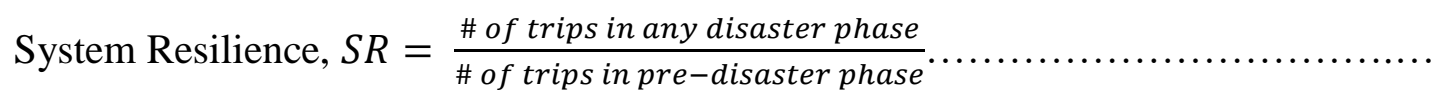

\subsection{Immediate Before Disaster Impact Phase (Phase 2, P2)}

In case of any forecasted disaster, the performance of transportation system in the immediate before disaster impact phase is critical and crucial for evacuation, response planning and resource allocation. Before the landfall of a forecasted disaster, the weather degrades significantly. For example, heavy rainfall and excessive wind (before any disaster) can cause significant performance reduction of transportation system. This excessive weather disruption phase before any disaster is defined here as the immediate before disaster impact phase. Usually, this phase can vary from few hours to few days based on the strength and type of the disaster. As during this phase, the performance of transportation system is critical due to the preparatory activities, the quantification of performance degradation during the phase is important. With the deployment of CAV in mixed-traffic scenario, the performance of system can be estimated through real time information from CAV vehicles. Using the equations from 1 to 10 , the performance metrics and resilience of the system can be estimated in immediate before disaster impact phase.

\subsection{Disaster Impact Phase (Phase 3, P3)}

Disaster impact phase starts at the onset of the disaster (for example, landfall of the hurricane). It may extend from few hours to a few days based on the disaster impacted area size and the strength of the disaster. During this phase, the transportation system performs at its worst level. To represent this performance, the resilience parameter of the system can go to the lowest value (even as of zero). The affected zone could be flooded or inoperable for a significant amount of time. Because 
of the limited traffic demand and near-zero performance during this phase, the researchers did not include this phase in the resilience analysis.

\subsection{Immediate After Disaster Impact Phase (Phase 4, P4)}

During the immediate after disaster impact phase (P4), the transportation system starts to operate on a limited scale. This phase ranges from the end of disaster impact phase to the start of the recovery and retrofitting activities. In this phase, the critical damage information such as number of dysfunctional links and nodes and partially or completely failure of CAV infrastructures is detected which are important to determine resource requirements to recover the system's resiliency in recovery phase (P5). By using equation 10 in preparation phase and in this phase and taking the difference of the values of the resilience parameter, the degradation in resilience of the system can be measured.

\subsection{Recovery Phase (Phase 5, P5)}

Recovery phase is defined as the completion of the recovery activities. After the recovery activities are completed, the resilience of the transportation system improves towards the pre-disaster condition. If the budget, resources, and recovery time are not enough to comprehend the type and extent of the damage, the system can lack in terms of the performance compared to pre-disaster scenario. The amount of the resiliency achieved after the recovery phase is critical in preparing for future disaster scenarios. Unfortunately, recovery efforts are usually constrained by public and private budgets, $B$ and recovery time $T_{r}$. If the system's damage state cannot be fully repaired within the budget and recovery time, then the post-disaster performance of the system would be lower than the pre-disaster. This method measured $B$ and $T_{r}$ using equations 11 and 12.

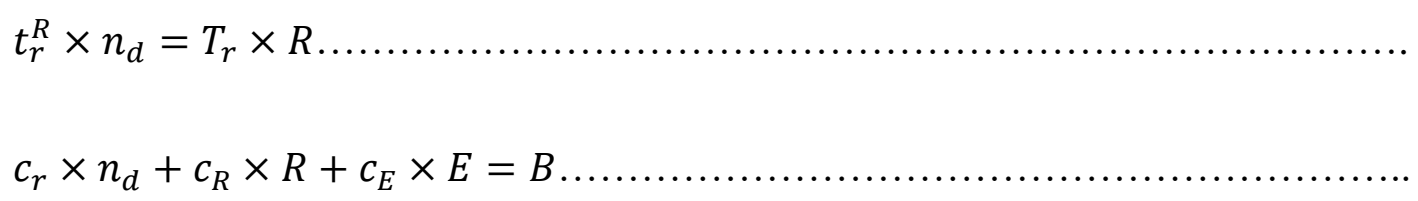

Where,

$t_{r}^{R}=$ Unit recovery time (in hours) needed for a repair team to repair a damaged unit

$n_{d}=$ Number of damaged units that are needed to be repaired 
$R=$ Number of repair teams needed

$E=$ Number of equipment (resources) needed

$c_{r}=$ Unit cost of repairing a damaged unit (in US \$)

$c_{R}=$ Unit cost of assigning a repair team (in US \$)

$c_{E}=$ Unit cost of using an equipment (in US \$) 


\section{CHAPTER 4: DISASTER SCENARIO AND NETWORK SYSTEM}

\subsection{Development of Disaster Scenarios}

To consider different scale of disasters, three scenarios (i.e., small-scale, medium-scale, and largescale) were considered in this research in which number of disrupted or failed nodes ranges from two to six (with an increment of two) from small-scale to large-scale disaster scenario (shown in Figure 2). The system resilience and other performance metrics were analyzed for P2, P4, and P5 for each disaster scenario. The change in route selection due to the failure of the nodes and links were considered in the system resilience calculation. Furthermore, as drivers' behavior change during abrupt weather situation such as increase in headway, a headway variation due to non-ideal roadway weather situation due to disaster was assumed in each phase based on the analysis from [45]. In general, due to the bad weather situation (e.g., heavy rainfall or excessive wind), some trips got canceled or postponed [46]. To accommodate this estimation, a portion of traffic demand reduction was assumed in each scenario. According to Hranac et al. [47], during adverse weather impact, traffic flow can be reduced by $10 \%$ to $30 \%$, while capacity can be reduced by $15 \%$ to $30 \%$ and speed can be reduced by 3-4 mph. Even, due to small scale pavement damage (partial failure condition), the congestion may occur on affected links and lead to reduction in traffic flow.

To comprehend all the above-mentioned typical traffic characteristics during disasters, we assumed (based on the estimation of the analysis of $(45,46,47))$ that traffic average headway increased by $3 \%$ in small scale, by $5 \%$ in medium scale, and by $10 \%$ in large scale in P2, while headway increment was considered as $10 \%, 15 \%$, and $25 \%$ respectively in $\mathrm{P} 4$. As in recovery phase, the abrupt weather situation is considered to be over, it was assumed that traffic will get back to typical headway scenario in P5 (i.e., recovery phase). In the case of traffic flow, the route preference and the availability of alternate routes were analyzed considering the impact of the failed nodes and links. Further a reduction in traffic demand as of, 15\% (in small scale disaster), 25\% (medium scale disaster), and 35\% (large-scale disaster) were considered to comprehend the travelers' disinterest to make trips, the congestion, abrupt weather condition, and partial smallscale damage on the links. 


\subsection{Transportation Network System}

The proposed resilience model was applied to measure the resilience of the Nguyen-Dupuis network system (Figure 4.1). Traditionally, this hypothetical network was considered as one-way network system in several transportation research studies. In this research, two-way movement was considered to reasonably represent a typical transportation network. Three highlighted corridors were assumed as arterials (Figure 4.1) and other links were assumed as collectors/local streets to consider a functional variation in the network. A speed limit of $45 \mathrm{mph}$ and two lanes in each direction was assumed for arterials and $25 \mathrm{mph}$ speed limit and one lane in each direction was assumed for collectors. O-D nodes are marked in the figure 4.1 with light blue color. Table 1 and 2 represents the network parameters in traditional traffic scenario which were collected and factorized from [47]. As [47] considered two-way traffic movement in the Nguyen-Dupuis network system for the analysis, this study adopted this network for the reliability of the network performance parameter. The resilience analysis was done for $0 \%, 25 \%, 50 \%, 75 \%$, and $100 \% \mathrm{CAV}$ penetration rates. Furthermore, Figure 4.2 - 4.4 shows the damaged nodes and links considered in different disaster-scenarios in P4.

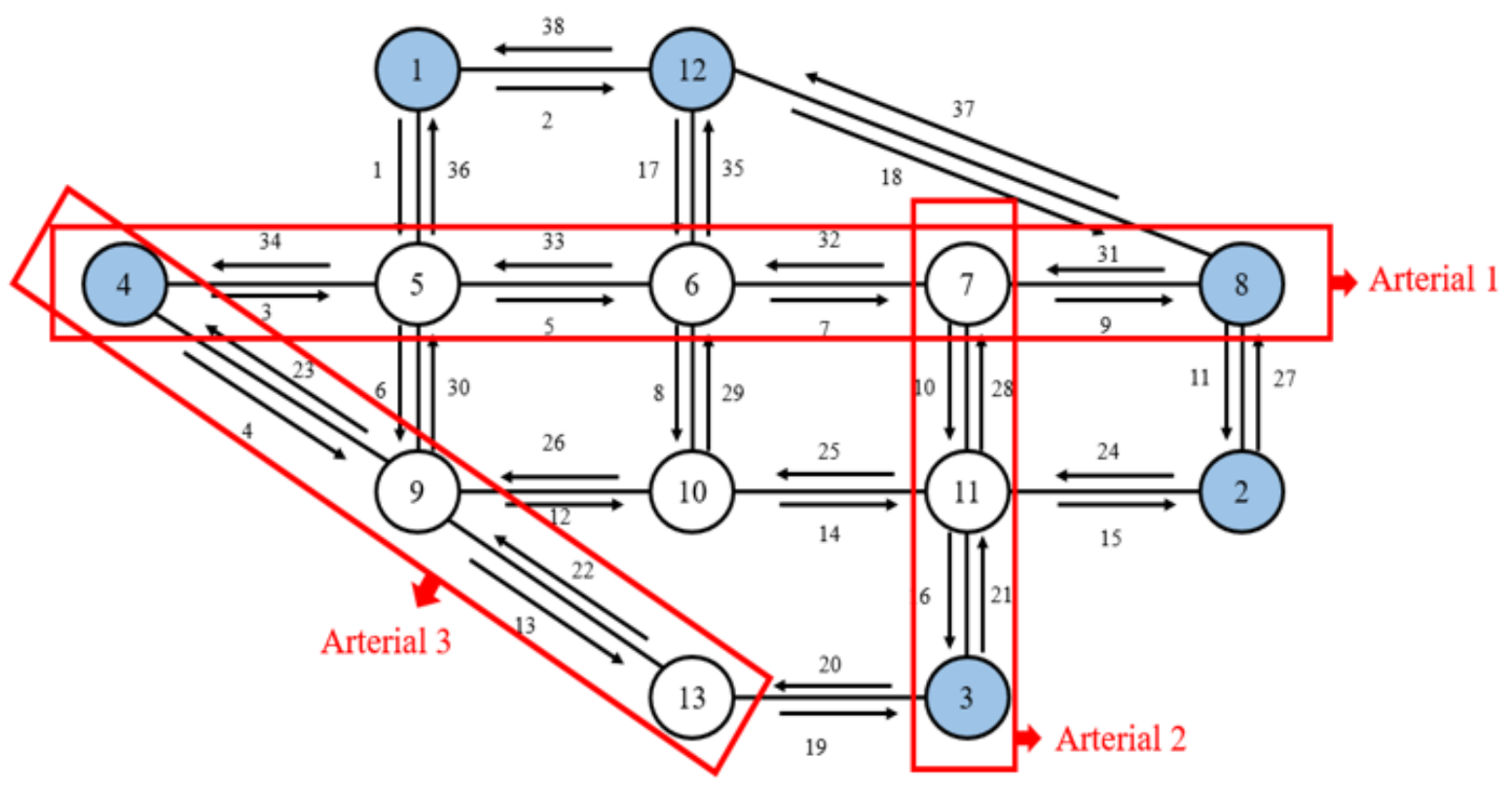

Figure 4.1: Nguyen-Dupuis Network (adopted from [47]) 


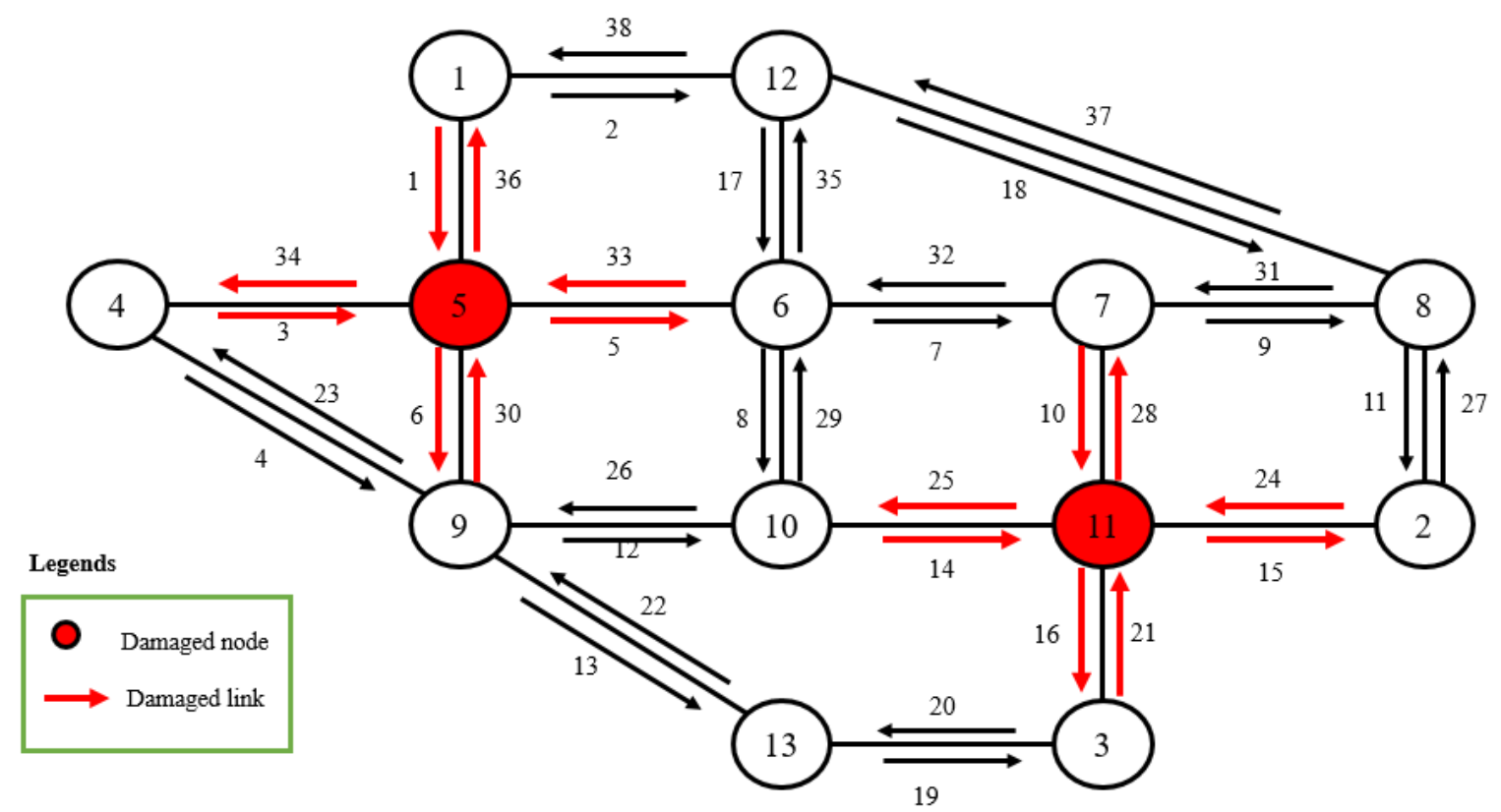

Figure 4.2: Damaged nodes and links considered in P4 resilience analysis small-scale disaster scenario

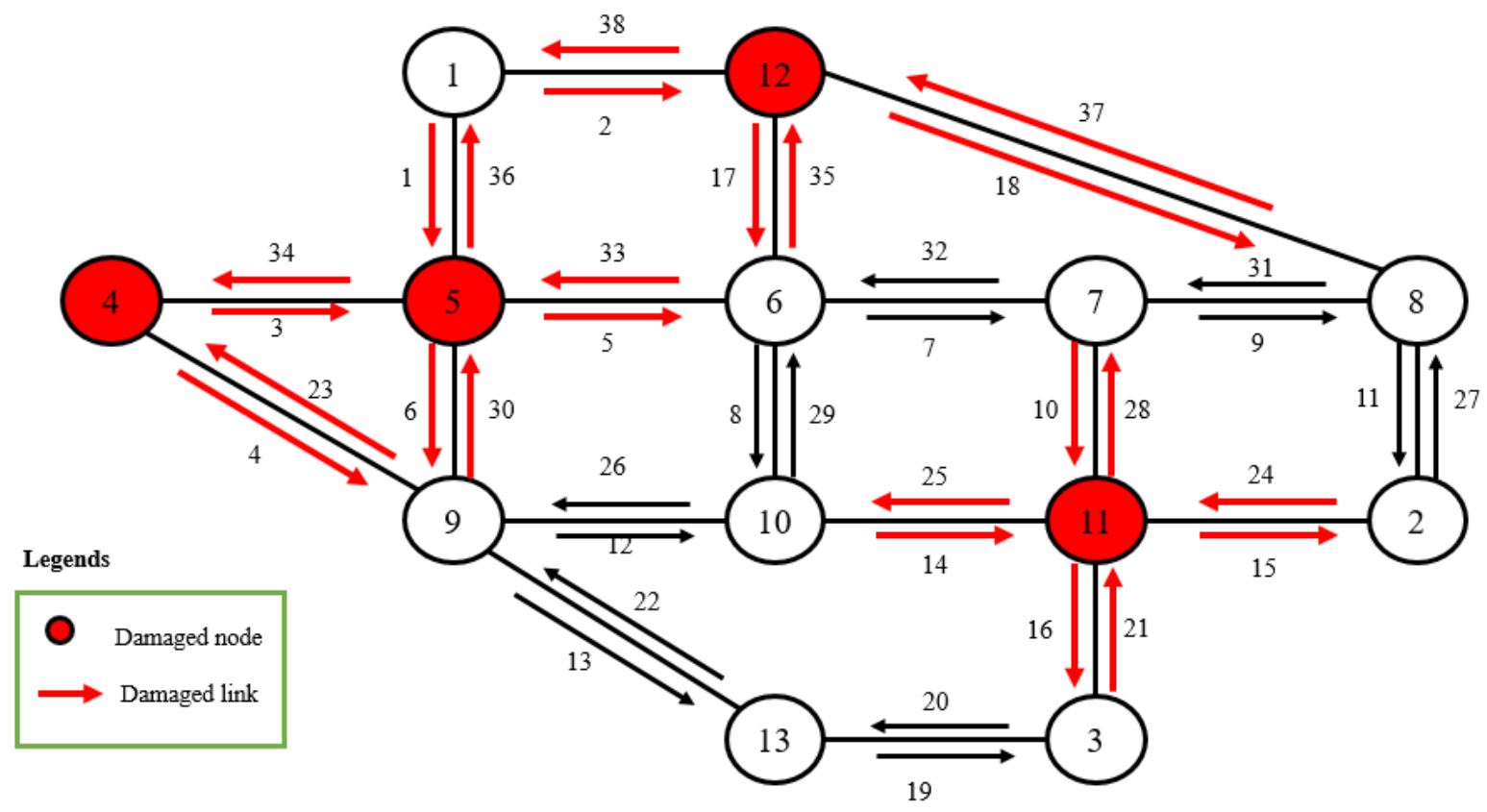

Figure 4.3: Damaged nodes and links considered in P4 resilience analysis in medium-scale disaster scenario 


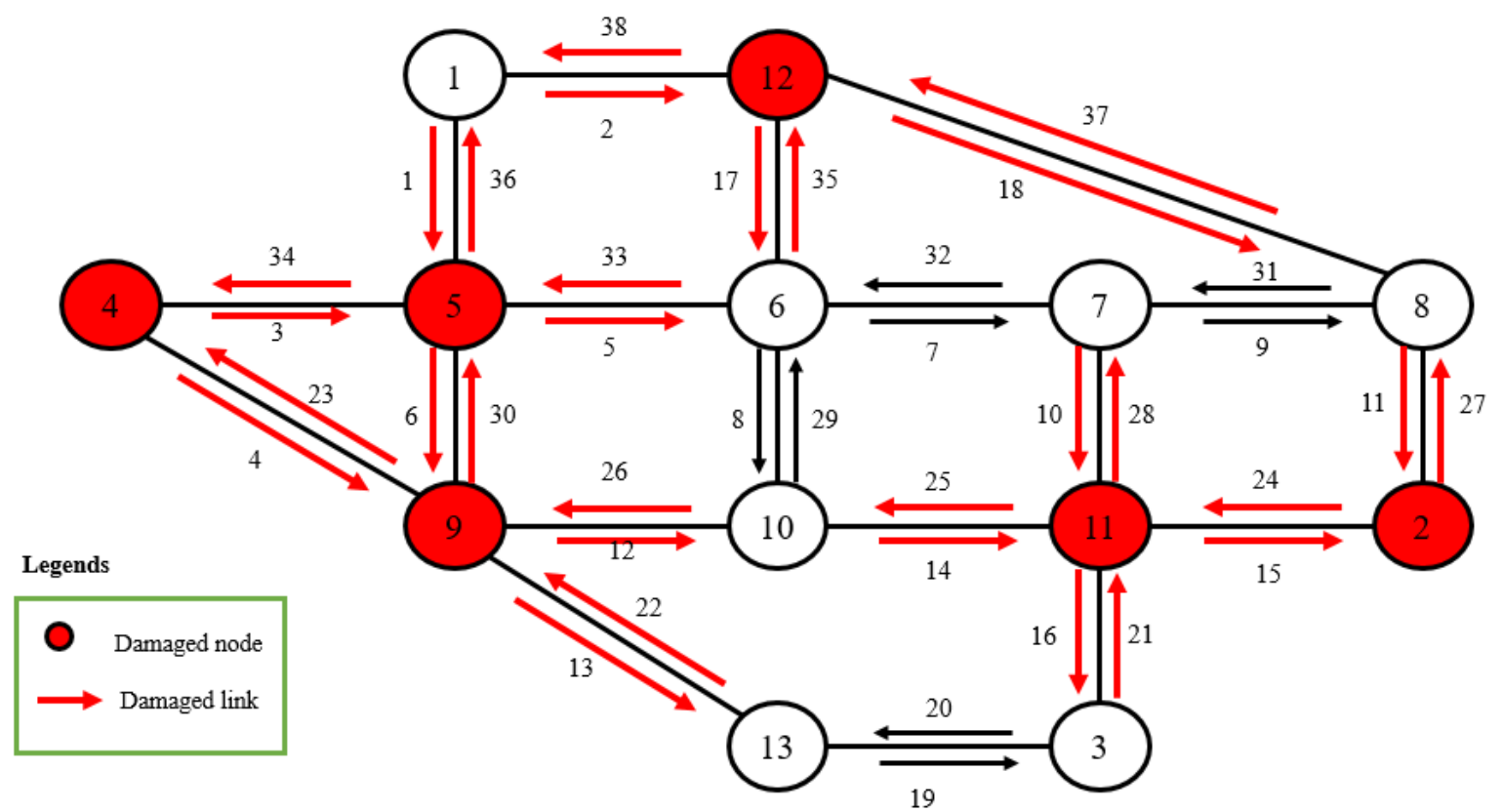

Figure 4.4: Damaged nodes and links considered in $\mathbf{P 4}$ resilience analysis in large-scale disaster scenario

Table 4.1: Given performance parameters for Nguyen Dupuis network for pre-disaster condition for manual vehicles (adopted from [47])

\begin{tabular}{|c|c|c|c|c|c|c|c|}
\hline $\begin{array}{c}\text { Link } \\
\#\end{array}$ & $\begin{array}{c}\text { Capacity } \\
(\mathbf{v} / \mathbf{h r} / \mathbf{l n})\end{array}$ & $\begin{array}{c}\text { Speed } \\
\text { limit } \\
(\mathbf{m p h})\end{array}$ & $\begin{array}{c}\text { FFTT } \\
(\mathbf{m i n})\end{array}$ & $\begin{array}{c}\text { Length } \\
(\mathbf{m})\end{array}$ & $\begin{array}{c}\text { Average } \\
\text { Velocity (mph) }\end{array}$ & $\begin{array}{c}\text { Flow } \\
(\mathbf{v} / \mathbf{h r} / \mathbf{l n})\end{array}$ & $\begin{array}{c}\text { Travel } \\
\text { time } \\
(\mathbf{m i n})\end{array}$ \\
\hline 1 & 2000 & 25 & 7 & 2.92 & 12.82 & 1900 & 13.65 \\
\hline 2 & 2000 & 25 & 9 & 3.75 & 13.37 & 1740 & 16.83 \\
\hline 3 & 1760 & 45 & 9 & 6.75 & 33.14 & 630 & 12.22 \\
\hline 4 & 1760 & 45 & 12 & 9.00 & 25.97 & 1290 & 20.80 \\
\hline 5 & 1760 & 45 & 3 & 2.25 & 24.44 & 1480 & 5.52 \\
\hline 6 & 2000 & 25 & 9 & 3.75 & 21.83 & 290 & 10.31 \\
\hline 7 & 1760 & 45 & 5 & 3.75 & 24.44 & 1480 & 9.20 \\
\hline 8 & 2000 & 25 & 13 & 5.42 & 22.03 & 270 & 14.76 \\
\hline 9 & 1760 & 45 & 5 & 3.75 & 35.36 & 480 & 6.36 \\
\hline 10 & 1760 & 45 & 9 & 6.75 & 28.70 & 1000 & 14.11 \\
\hline
\end{tabular}




\begin{tabular}{|c|c|c|c|c|c|c|c|}
11 & 2000 & 25 & 9 & 3.75 & 15.97 & 1130 & 14.09 \\
\hline 12 & 2000 & 25 & 10 & 4.17 & 16.89 & 960 & 14.80 \\
\hline 13 & 1760 & 45 & 9 & 6.75 & 33.28 & 620 & 12.17 \\
\hline 14 & 2000 & 25 & 6 & 2.50 & 15.48 & 1230 & 9.69 \\
\hline 15 & 2000 & 25 & 9 & 3.75 & 16.08 & 1110 & 14.00 \\
\hline 16 & 1760 & 45 & 8 & 6.00 & 27.50 & 1120 & 13.09 \\
\hline 17 & 2000 & 25 & 7 & 2.92 & 22.03 & 270 & 7.95 \\
\hline 18 & 2000 & 25 & 14 & 5.83 & 12.76 & 1920 & 27.44 \\
\hline 19 & 2000 & 25 & 11 & 4.58 & 19.08 & 620 & 14.41 \\
\hline 20 & 2000 & 25 & 11 & 4.58 & 13.81 & 1620 & 19.91 \\
\hline 21 & 1760 & 45 & 8 & 6.00 & 42.13 & 120 & 8.55 \\
\hline 22 & 1760 & 45 & 9 & 6.75 & 23.43 & 1620 & 17.28 \\
\hline 23 & 1760 & 45 & 12 & 9.00 & 30.58 & 830 & 17.66 \\
\hline 24 & 2000 & 25 & 9 & 3.75 & 20.00 & 500 & 11.25 \\
\hline 25 & 2000 & 25 & 6 & 2.50 & 19.53 & 560 & 7.68 \\
\hline 26 & 2000 & 25 & 10 & 4.17 & 20.00 & 500 & 12.50 \\
\hline 27 & 2000 & 25 & 9 & 3.75 & 13.37 & 1740 & 16.83 \\
\hline 28 & 2000 & 25 & 9 & 3.75 & 24.27 & 60 & 9.27 \\
\hline 29 & 2000 & 25 & 13 & 5.42 & 24.27 & 60 & 13.39 \\
\hline 30 & 2000 & 25 & 9 & 3.75 & 15.20 & 1290 & 14.81 \\
\hline 31 & 1760 & 45 & 5 & 3.75 & 28.09 & 1060 & 8.01 \\
\hline 32 & 1760 & 45 & 5 & 3.75 & 27.50 & 1120 & 8.18 \\
\hline 33 & 1760 & 45 & 3 & 2.25 & 28.09 & 1060 & 4.81 \\
\hline 34 & 1760 & 45 & 9 & 6.75 & 39.88 & 226 & 10.16 \\
\hline 35 & 2000 & 25 & 7 & 2.92 & 23.58 & 120 & 7.42 \\
\hline 36 & 2000 & 25 & 7 & 2.92 & 12.44 & 2020 & 14.07 \\
\hline 37 & 2000 & 25 & 14 & 5.83 & 12.66 & 1950 & 27.65 \\
\hline 38 & 2000 & 25 & 9 & 3.75 & 13.81 & 1620 & 16.29 \\
\hline
\end{tabular}


Table 4.2: Origin-Destination (OD) distribution used in Nguyen Dupuis network

\begin{tabular}{|c|c|c|c|}
\hline OD pair \# & Origin $(\mathbf{O})$ & Destination (D) & Flow $\left(f_{O D}^{t_{i}}\right)$ \\
\hline 1 & 1 & 2 & 630 \\
\hline 2 & 1 & 3 & 1290 \\
\hline 3 & 1 & 8 & 960 \\
\hline 4 & 2 & 1 & 630 \\
\hline 5 & 2 & 4 & 960 \\
\hline 6 & 2 & 12 & 150 \\
\hline 7 & 3 & 1 & 1290 \\
\hline 8 & 3 & 4 & 330 \\
\hline 9 & 3 & 12 & 120 \\
\hline 10 & 4 & 2 & 960 \\
\hline 11 & 4 & 3 & 330 \\
\hline 12 & 4 & 8 & 630 \\
\hline 13 & 8 & 1 & 960 \\
\hline 14 & 8 & 4 & 630 \\
\hline 15 & 8 & 12 & 180 \\
\hline 16 & 12 & 2 & 150 \\
\hline 17 & 12 & 3 & 120 \\
\hline 18 & 12 & 8 & 180 \\
\hline
\end{tabular}




\section{CHAPTER 5: RESULTS AND DISCUSSION}

\subsection{Disruption Impact Factor (DIF) Variation for Nodes}

Based on different weights assigned to components of DIF (i.e., connected links length, speed and flow characteristics), DIF ranking of a node can vary. Rank 1 (i.e., maximum DIF value) represents the most critical node in the network whose failure may affect the performance of the network most. From the analysis, it was evident that depending on the relative importance given on the three parameters, the criticality (ranking) of nodes could change. The rationale behind this phenomenon is that some nodes are more flow sensitive (means they serve higher traffic flow) or some are speed sensitive (i.e., higher speed). As a result, if larger weight is assigned to flow, higher traffic flow serving nodes become most critical nodes, whereas, if average speed is assigned larger weight, nodes connected to high speed links become more critical. For example, node 4 was connected to high speed links. When the average flow parameter was given equal weight or larger compared to average speed and link length (Combination 1, 2, and 3), the ranking of node 4 was 3. However, when the speed was assigned more weight compared to flow (Combination 4), the criticality of node 4 increased (from rank 3 to 2). In this work, DIF was calculated based on four weightage combinations and finally, DIF of nodes was considered based on the average of those four combinations. DIF rank of nodes for four combinations of $\alpha, \beta$, and $\gamma$ and the average of four combinations is shown in Figure 5.1. It is evident from Figure 5.1 that node 8 is the most critical nodes as it is connected with neighborhood links that have the shortest link length, higher link flow, and higher link velocity. 


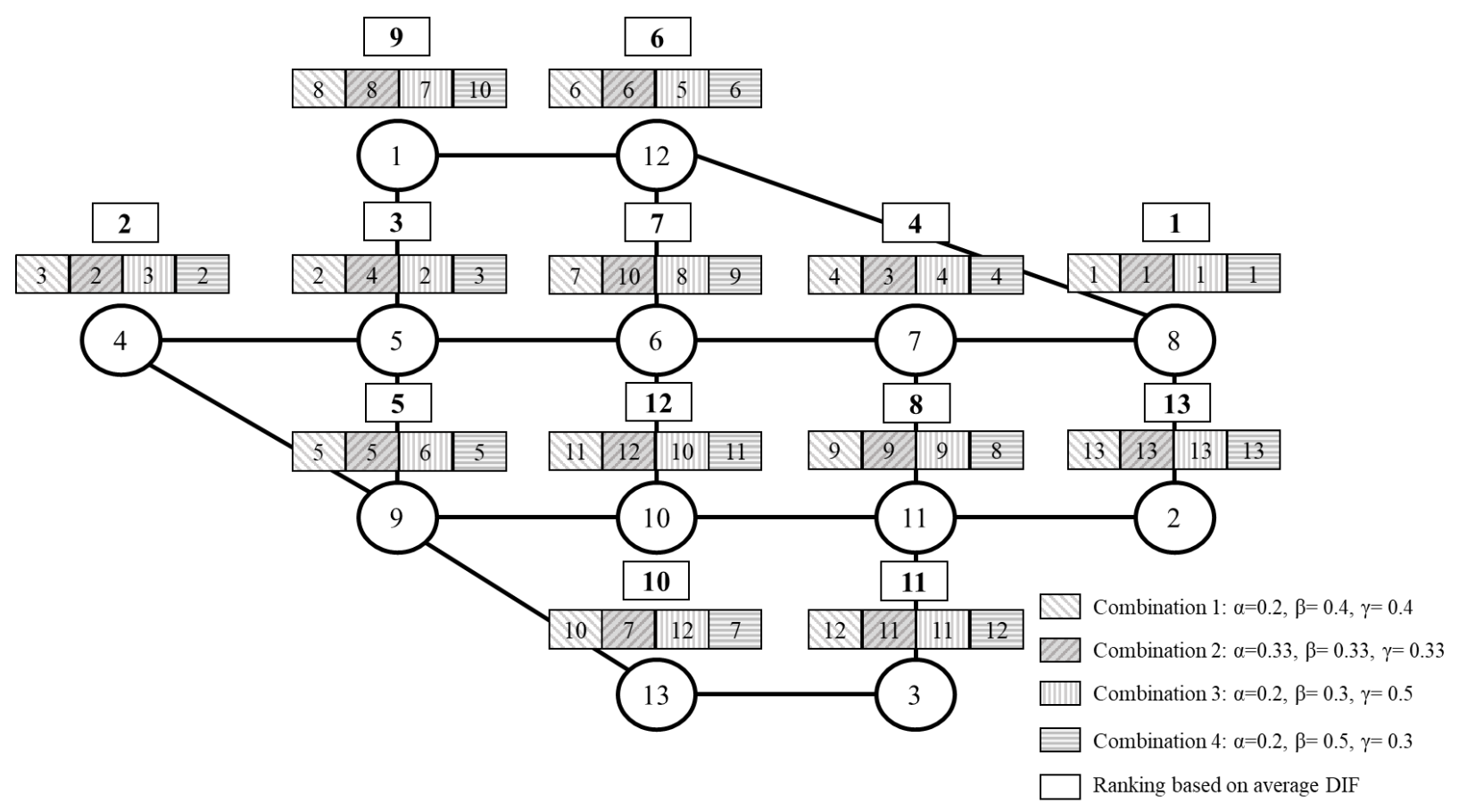

Figure 5.1: DIF ranking of nodes for different $\alpha, \beta$, and $\gamma$ combinations and average DIF ranking

\subsection{Network Capacity Variation}

The variation in terms of the network capacity to serve trips for different OD pairs are analyzed and shown in Figure 5.2. The analysis showed, the average capacity drop in P2 phase increased by disaster severity from small-scale to large-scale disaster scenario due to relative severe pre-disaster road weather conditions that lead to higher headway. In P4 phase the average capacity drop was increased by $40 \%$ (medium-scale) and 72\% (large-scale) and in P5 phase the average capacity drop was increased by $38 \%$ (medium-scale) and 62\% (large-scale). From these average capacity drops in P4 and P5, the relative improvement (in terms of capacity gain from node and link repair) for varied disaster scenarios with same budget and resource can be estimated. 


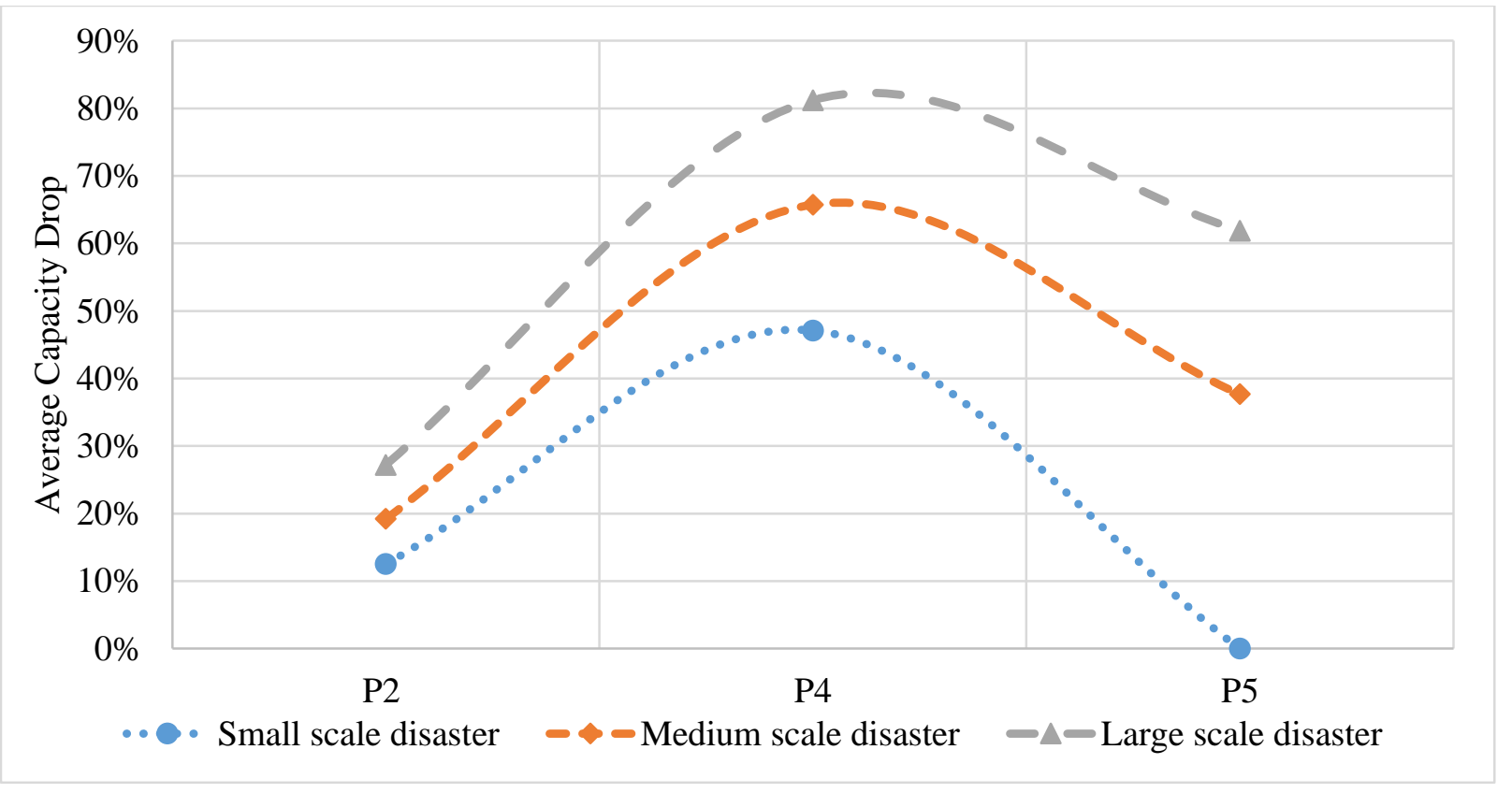

Figure 5.2: Average capacity drop in mixed-traffic environment for varied disaster scenarios

\subsection{Travel Time Variation}

Individual link travel time variation during different disaster phases are shown in Figures 5.3 to 5.9, and summarized in Figure 5.10. The key observation from the analysis are the following:

(i) Individual link travel time variation during different disaster phases followed similar trend with different CAV penetration rates. However, with the increasing penetration of $\mathrm{CAV}$, the travel time reduction was greater as large number of CAVs at higher penetration rates allowed smaller average headways (For example, link\# 18 in Figure 5.3-5.7). The results indicated that the travel time showed statistically significant reduction at $95 \%$ confidence with higher penetration of CAV in any disaster phase.

(ii) Individual link travel time value of 0 (zero) represented the failed links due to disaster and cannot be accessed during P4 phase until recovery activities restore the capacity of the affected links.

(iii) Individual link travel time increased for same CAV penetration in P2 phase with the increased severity of disaster scenario due to relatively severe bad weather condition in immediate before disaster impact phase (P2) (For example, link\# 18 in Figure 5.4, 5.8, 5.9).

(iv) Individual link travel time variation for varied disaster scenarios in P4 and P5 phases and varied CAV penetration dependents on the total number of failed components including nodes 
and links in each disaster scenario. If this number changes, travel time variation of un-affected or partially affected links will also change (For example, link\# 20 in Figure 5.4, 5.8, 5.9).

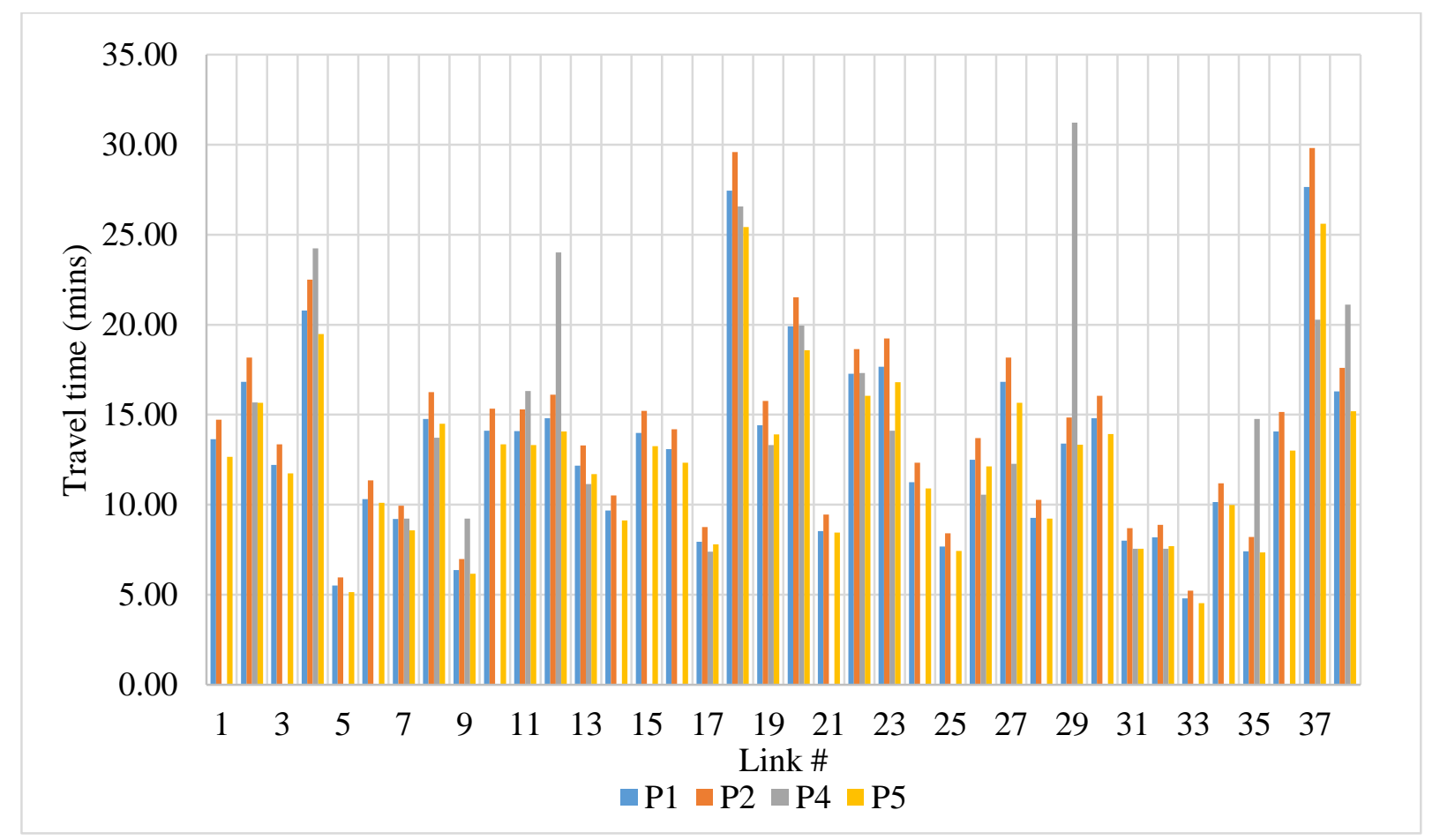

Figure 5.3: Individual link's travel time variation for 0\% CAV penetration due to the impact of the small-scale disaster scenario 


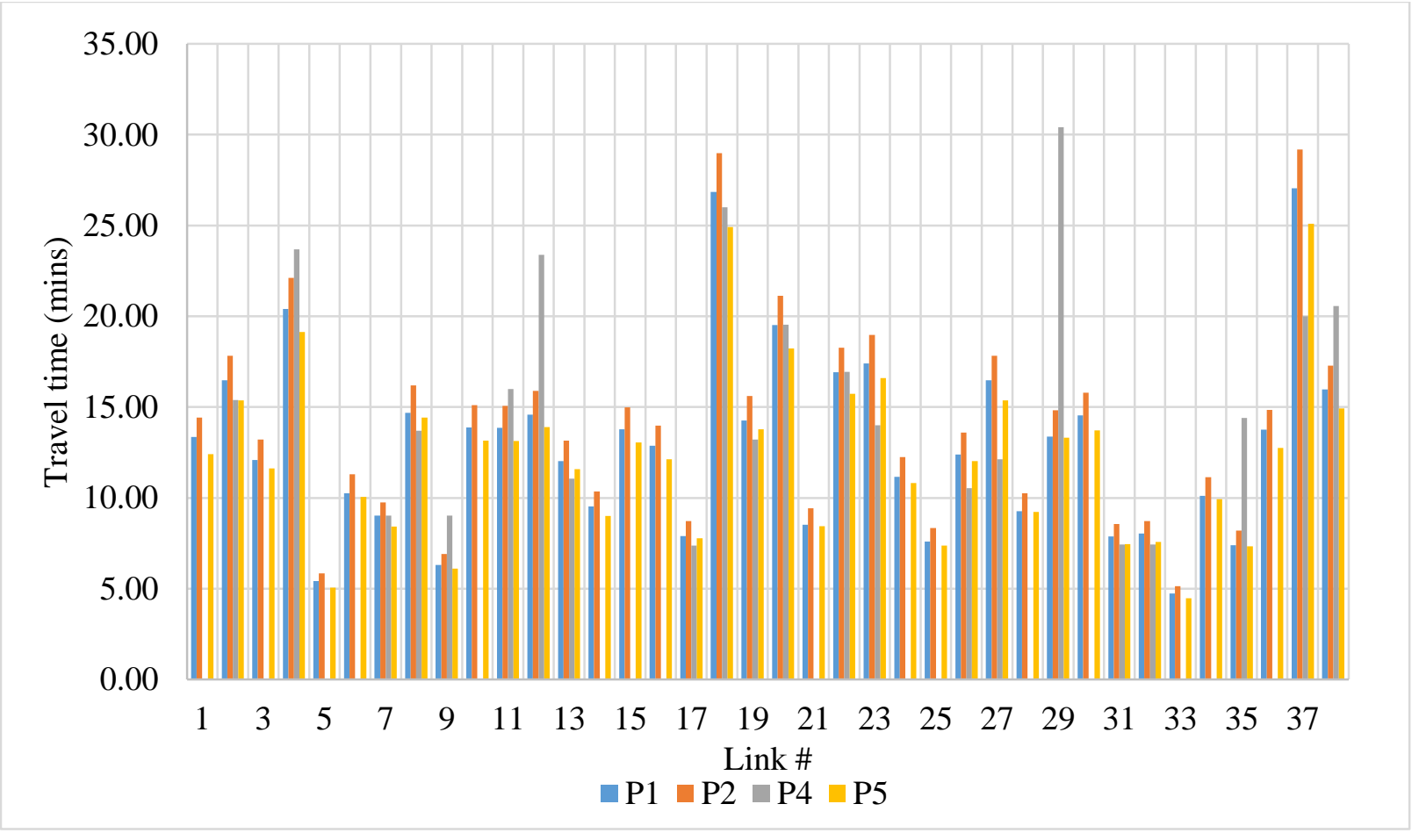

Figure 5.4: Individual link's travel time variation for $25 \%$ CAV penetration due to the impact of the small-scale disaster scenario

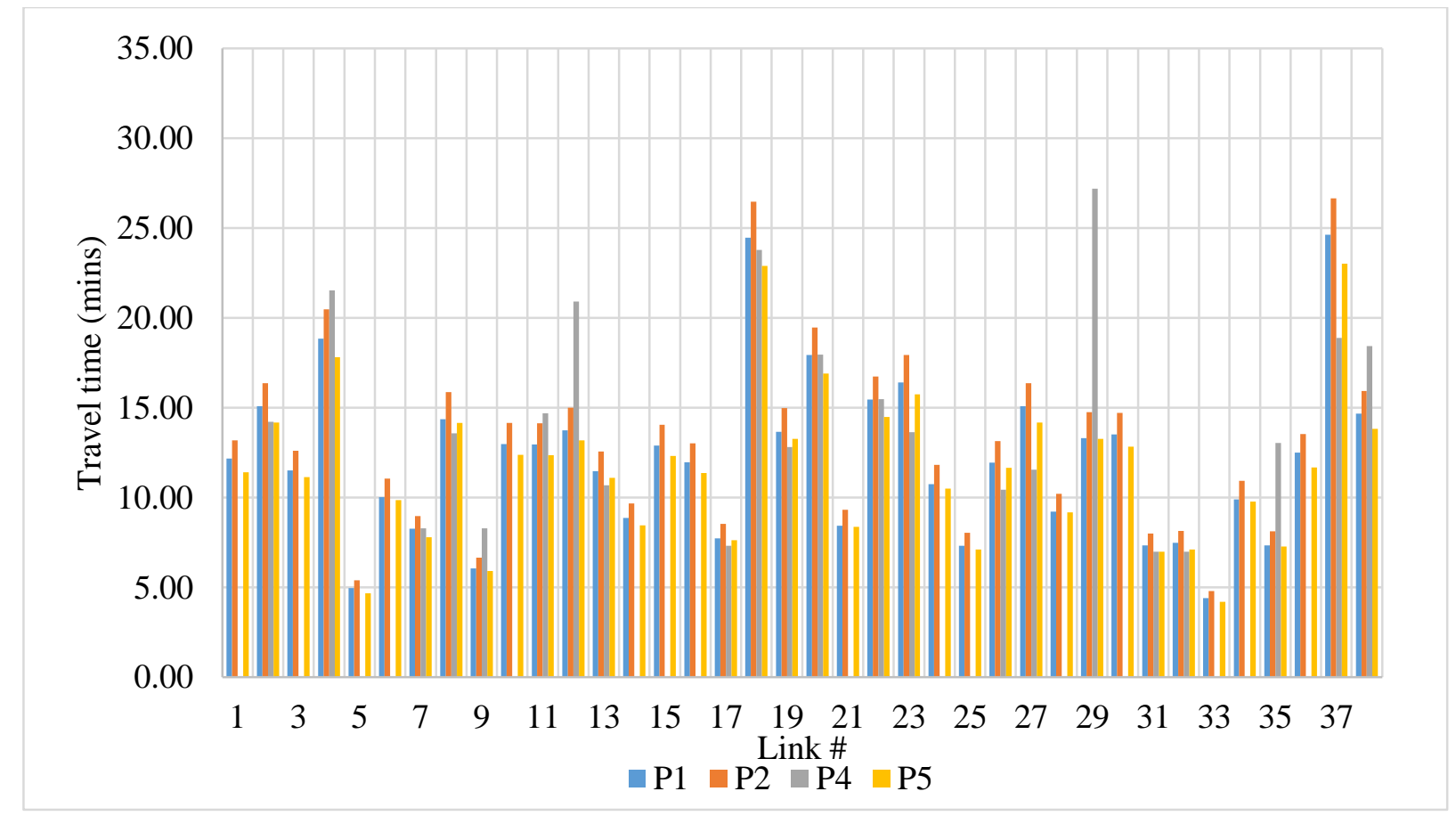

Figure 5.5: Individual link's travel time variation for 50\% CAV penetration due to the impact of the small-scale disaster scenario 


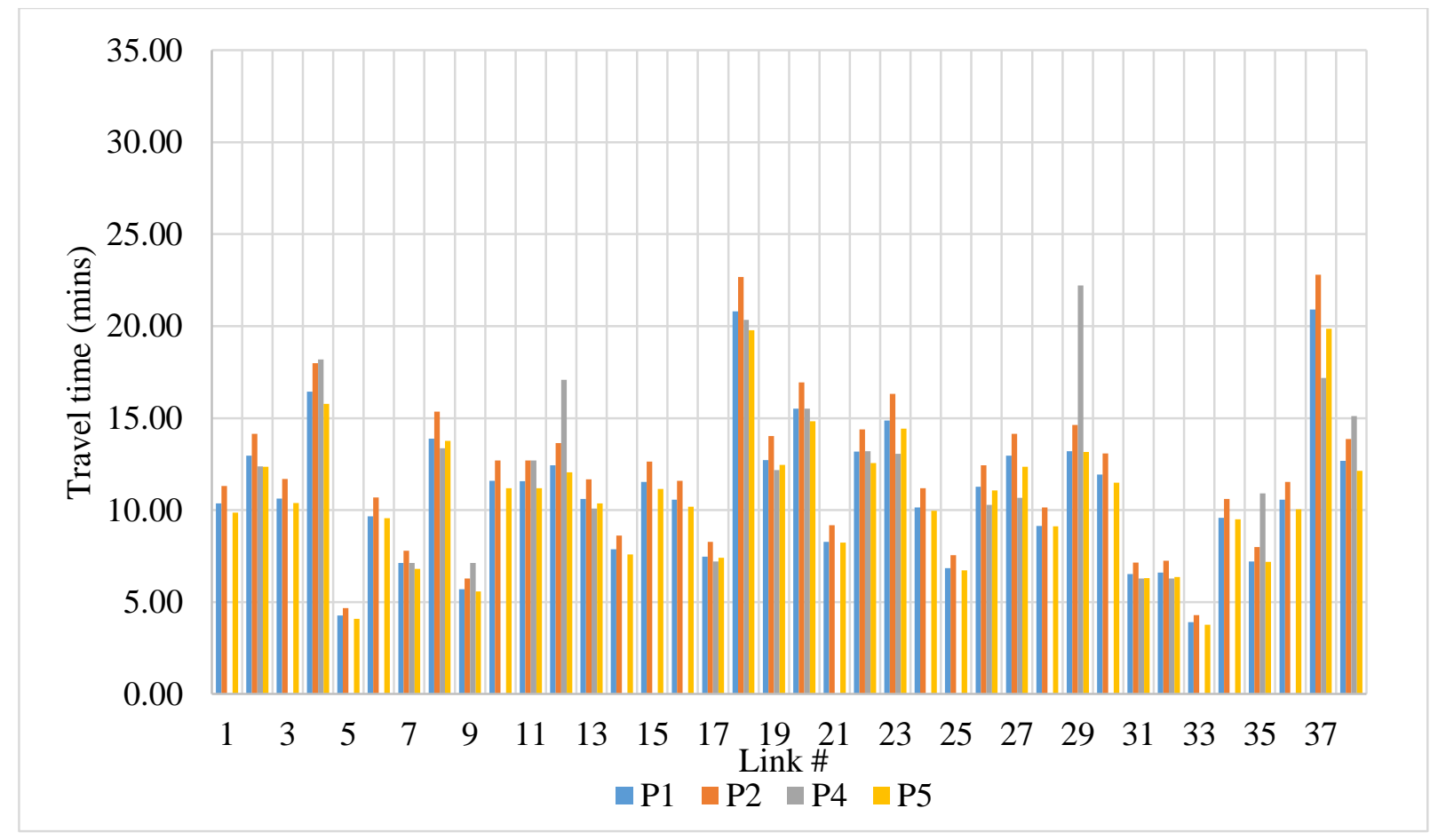

Figure 5.6: Individual link's travel time variation for $75 \%$ CAV penetration due to the impact of the small-scale disaster scenario

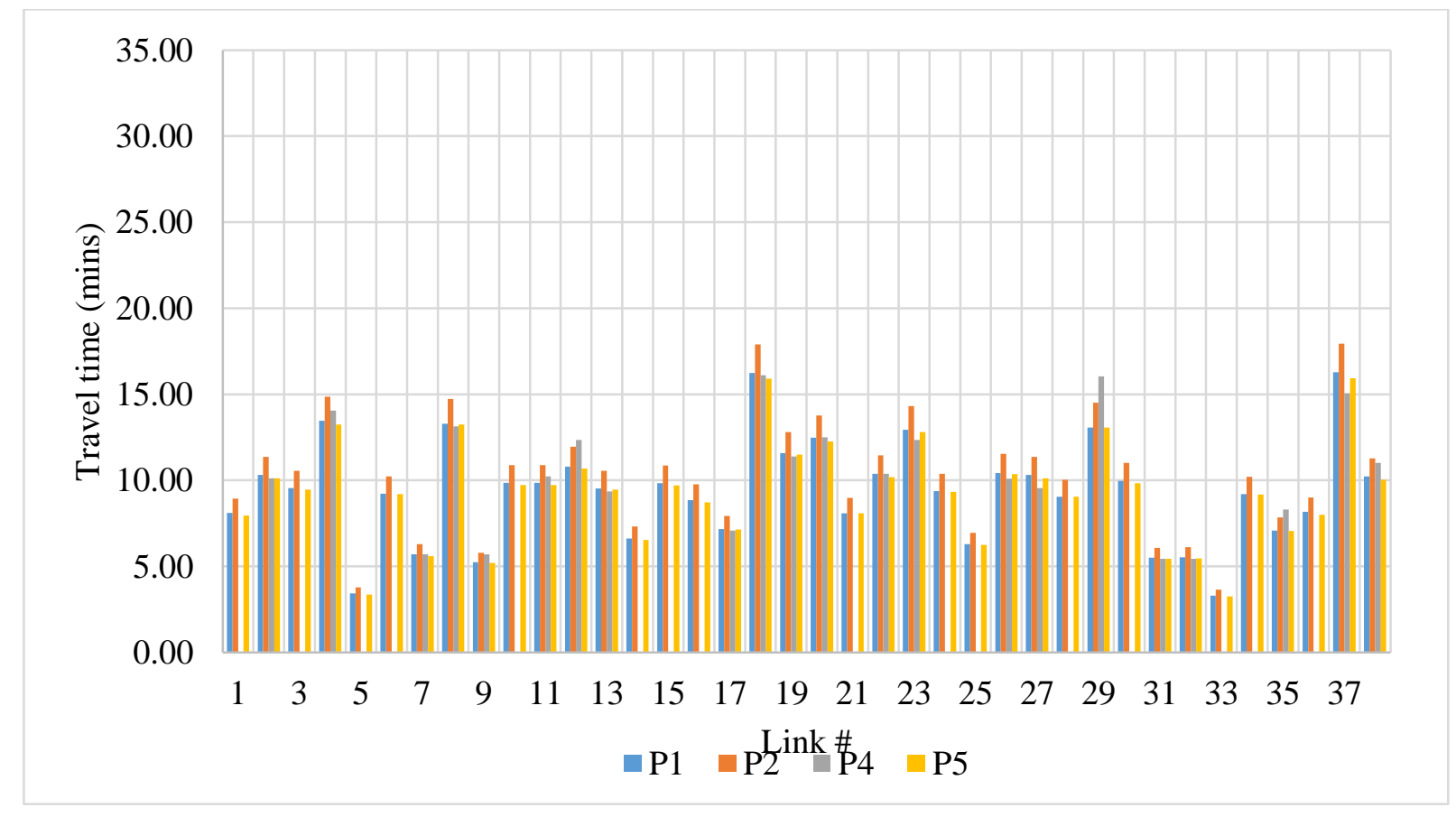

Figure 5.7: Individual link's travel time variation for $100 \%$ CAV penetration due to the impact of the small-scale disaster scenario 


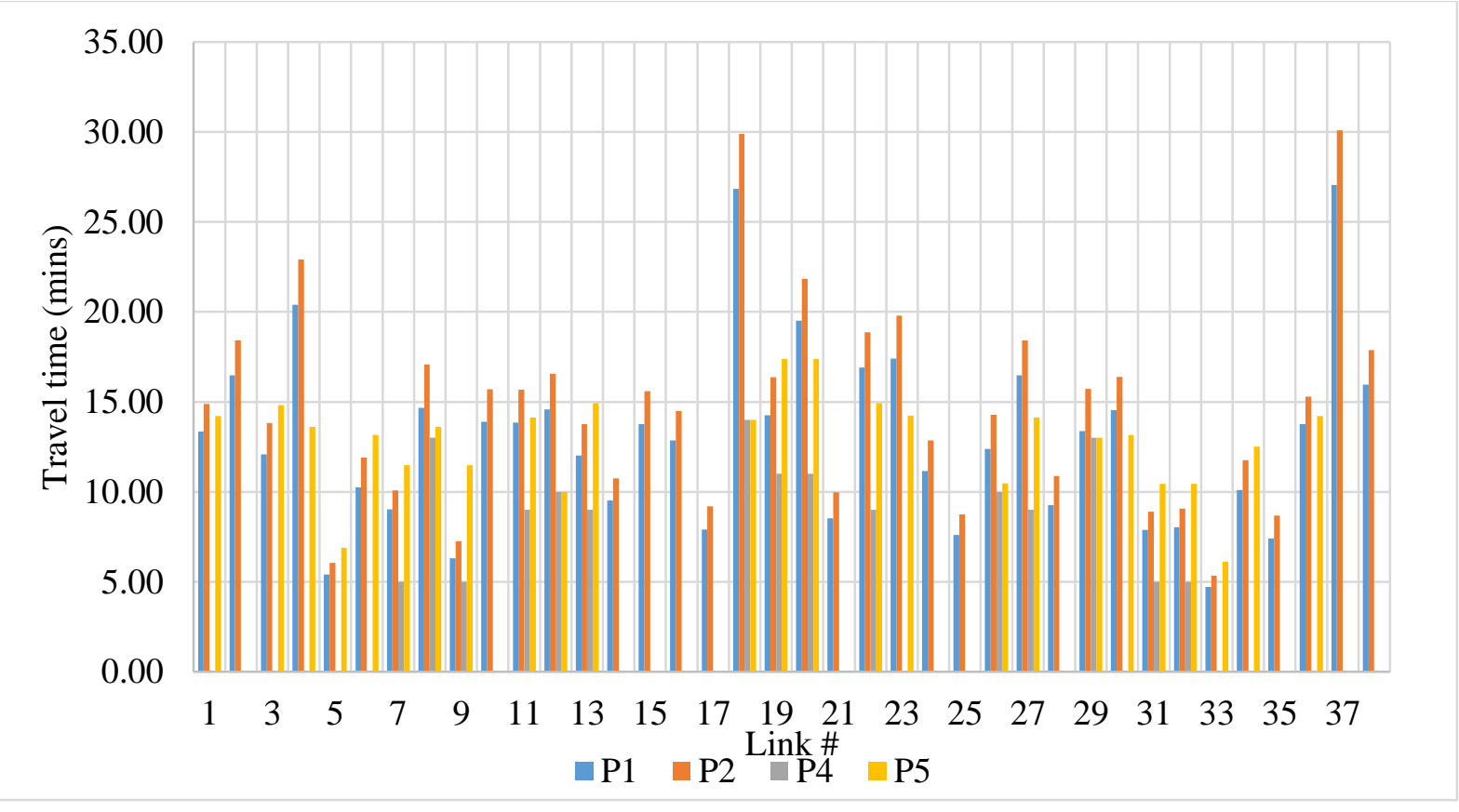

Figure 5.8: Individual link's travel time variation for medium-scale disaster scenario for 25\% CAV penetration

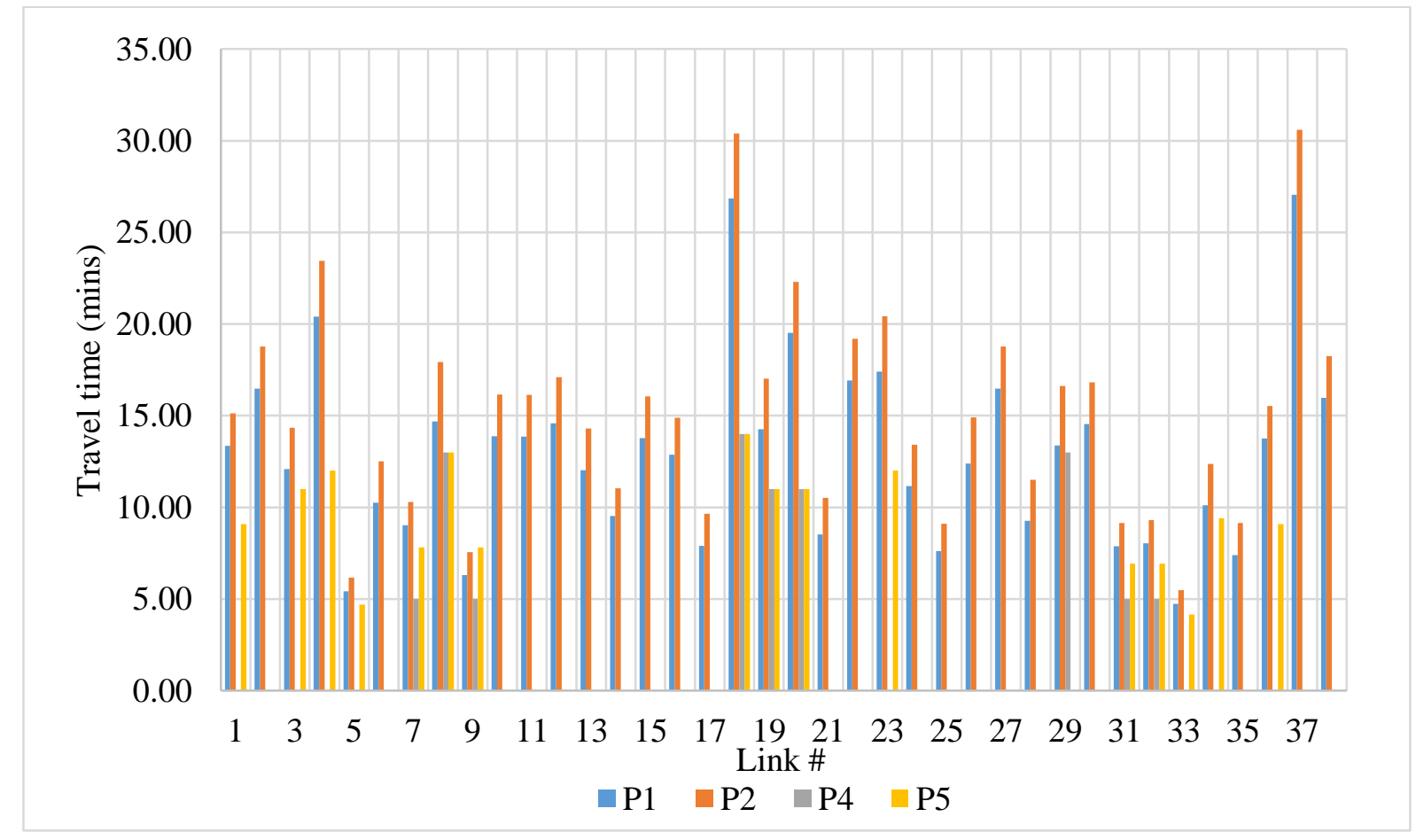

Figure 5.9: Individual link's travel time variation for large-scale disaster scenario for $25 \%$ CAV penetration 
Further, in this section, the percentage increment in link travel time in P4 was compared with link travel time in no-CAV (0\%), no-disaster scenario. The maximum, average, and minimum increment in link travel time due to the impact of small-scale disaster scenario was presented in the Figure 5.10.

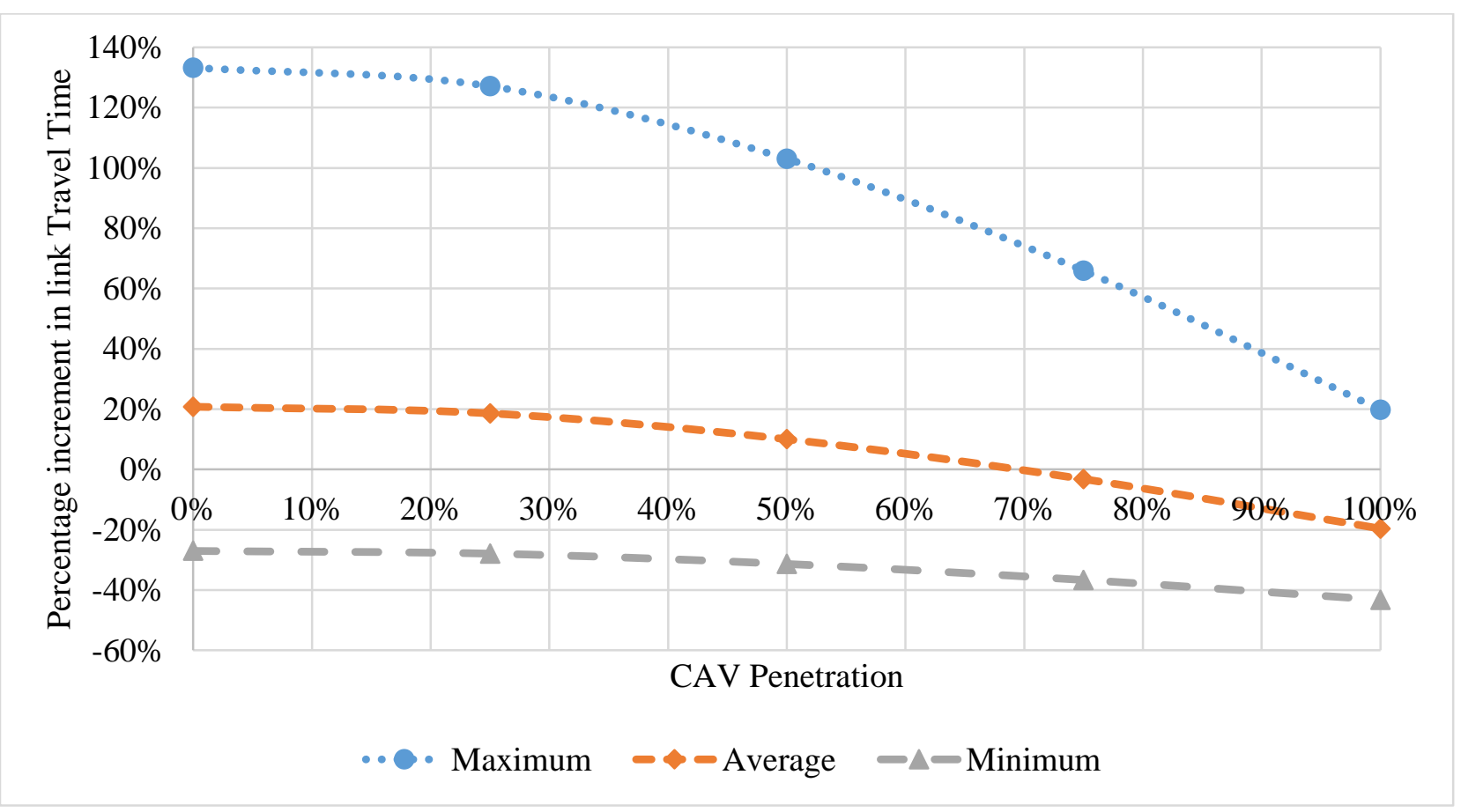

Figure 5.10: Percentage increase in link travel time in $P 4$ phase in small-scale disaster scenario with different $\mathrm{CAV}$ penetration

In P4 phase, many pre-disaster shortest routes between OD pairs were damaged or became dysfunctional. Therefore, many trips must rely on limited available routes to complete trips. Due to this condition, the traffic flow on some links (e.g., link no. 29), increased significantly compared to the pre-disaster link traffic flow. This travel time analysis was done only for small-scale disaster scenario. In case of medium-scale and large-scale disasters, the impact of disasters in P4 phase was so severe that significant number of trips were not possible due to the failure of all available routes between many OD pairs. The key observations from this analysis are:

(i) On average, link travel time was reduced with higher CAV penetration. Maximum increment in link travel time in small-scale disaster scenario was observed on link no. 29. Due to the non-functionality of certain links due to disaster, the traffic flow in this link during P4 was almost 40 times higher than pre-disaster phase and, and travel time was increased by about $133 \%$. 
However, with higher CAV penetration (i.e., 100\% CAV penetration), this travel time increment can be reduced to $20 \%$ from maximum increment of $133 \%$.

(ii) Minimum increment in link travel time was observed on link no. 27 due to $62 \%$ reduction in link traffic flow compared to pre-disaster link traffic flow. This reduction in traffic flow was due to non-availability of alternate shortest routes that includes this link between certain OD pairs. With higher CAV penetration this travel time can be reached to $43 \%$ (at 100\% CAV penetration) compared to pre-disaster travel time.

\subsection{System Resilience (SR) Analysis}

As previously mentioned, during the pre-disaster phase, the transportation system resilience was assumed to be equal to 1 . The variation in system resilience due to the impact of the various disaster scenarios (discussed in Section 4.1) in different disaster phases is shown in Figure 5.15. Some other key observations from system resilience variation is in the following:

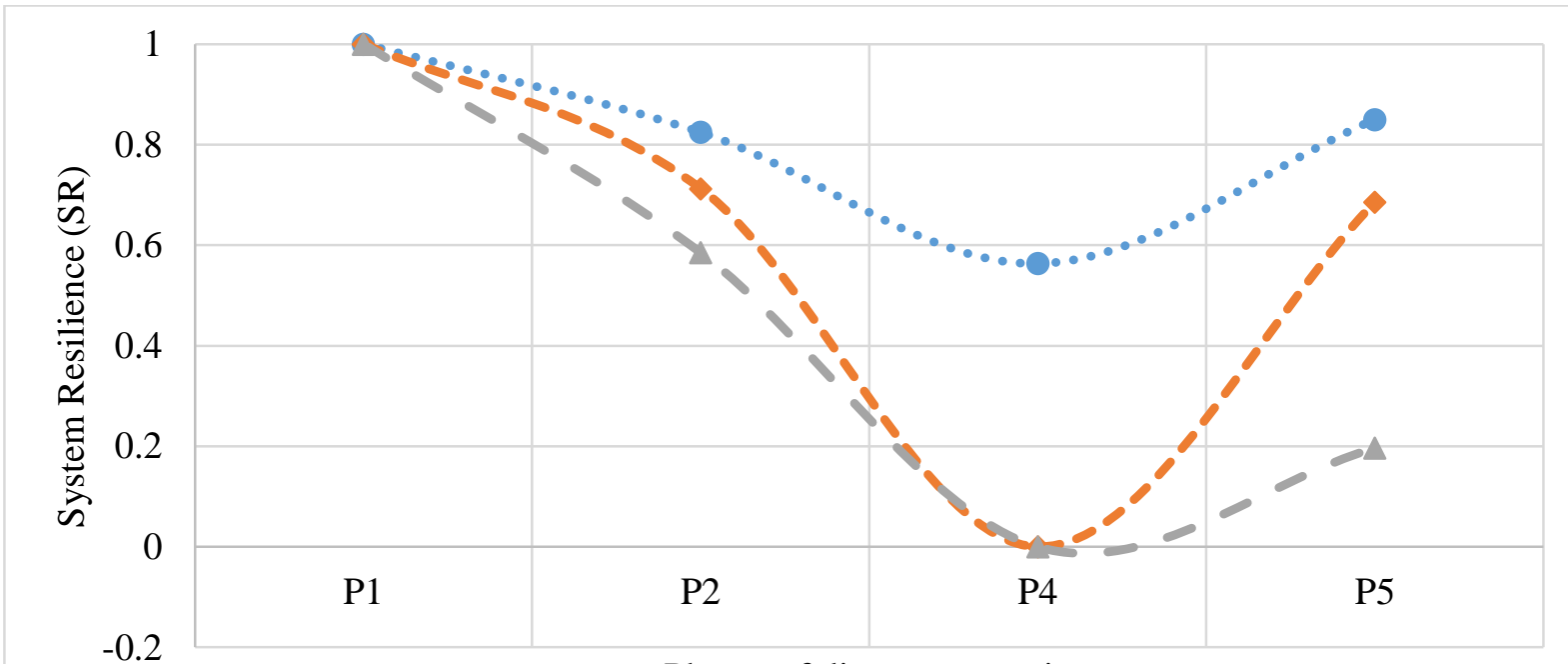

Phases of disaster scenario

$\cdots$ Small scale disaster $\quad-\diamond-$ Medium scale disaster $\quad-\triangle-$ Large scale disaster

Figure 5. 11: Transportation system resilience variation for different disaster scenarios

(i) SR followed a gradual decreasing trend in P2 in all the disaster scenarios compared to P1. The reduction of SR was $18 \%$ in small-scale disaster scenario, suggesting that $18 \%$ of the predisaster trips were disrupted due to the disaster impact. The medium and large-scale disaster scenarios were predicted to have a SR reduction of $29 \%$ and $41 \%$, respectively. This trend of SR 
in P2 followed the gradual increase in disaster intensity from small-scale to large-scale disaster scenario.

(ii) In P4, the total number of disrupted nodes and links was significantly greater in largescale than medium-scale disaster scenario. However, the SR value in both the scenarios was equal to zero, due to the complete failure (unavailability of routes) of all routes serving OD trips suggesting that even a comparatively low impact disaster can hamper all the routes for the hypothetical network used in this study.

(iii) The importance of recovery efforts can be explained through evaluating the SR value in P5. Here, similar recovery efforts (in terms of recovery time, recovery teams, and equipment) was considered and the maximum improvement in P5 was compared to P4. The improvement was found to be $69 \%$ for the medium-scale disaster scenario, $29 \%$ for the small-scale and $20 \%$ for the large-scale. Further investigation revealed that two of the most critical nodes (according to DIF ranking) were impacted during the medium- scale disaster scenario. Rapid repair of these two nodes was possible after the medium-scale disaster, therefore, the medium-scale disaster scenario showed the most improvement. On the other hand, during the large-scale disaster scenario, additional two critical nodes (total four nodes) were impacted (making the greatest reduction in SR in P4), but only two were recovered in P5 due to limited recovery budget.

When the impact of the disaster is large such as in large-scale disaster scenario, the number of damaged infrastructural components (links and nodes) will be higher. As CAVs significantly depend on the functional availability of the CAV infrastructural elements (e.g., road side unit for $\mathrm{V} 2 \mathrm{~V}$ and V2I communication), to restore the functionality of transportation system at pre-disaster

level, more repair teams and higher repair budget will be required. A comprehensive analysis of recovery budget and recovery time is explained in the next section.

\subsection{Analysis of Recovery Budget and Recovery Time}

The impacts of disaster preparedness activities in the pre-disaster phase (Phase 1) was not considered in the resilience analysis. Well planned pre-disaster management such as resource or equipment allocation, training of the recovery teams, estimating the impact of the upcoming disaster can improve the efficiency of the recovery activities. This study assumed equal preparedness (in terms of budget and resources) for all three disaster scenarios. 
To estimate improvement in the post-disaster performance of the transportation network, this study analyzed the required recovery budget and recovery time applying equations 11 and 12 in terms of the number of required recovery teams, recovery efforts (in terms of budget, equipment and recovery manpower) and affected number of nodes and links. Required recovery resources include recovery time and budget needed to repair all the damaged components in the small-scale disaster scenario as the base recovery time $\left(T_{r}\right)$ and base recovery budget $(\mathrm{B})$. With required small-scale recovery budget and recovery time, approximately $63 \%$ link and $85 \%$ node functionality (in terms of number) can be restored in medium-scale disaster scenario, and approximately $42 \%$ link and 69\% node functionality can be restored in the large-scale disaster scenario. Therefore, achieving $100 \%$ recovery in the post-disaster phase can be challenging if recovery time and budget are not adjusted accordingly. For lower penetration of CAVs, predominant non-CAVs can perform efficiently as these vehicles do not require CAV infrastructure support. Whereas for higher penetration of CAVs (>50\%), additional budget and resources are needed to repair the required infrastructure within recovery time.

Impact of recovery budget and recovery time were analyzed in this research. Total number of affected nodes and links were 18, 28, and 36 in the small-scale, medium-scale and large-scale disaster scenario, respectively. Therefore, with the same number of recovery teams, efforts and resources, the recovery time will be approximately 50\% longer after a medium-scale disaster scenario and $100 \%$ longer in large scale-disaster scenario, compared to the recovery time needed after a small-scale disaster scenario. This variation in recovery time greatly depends on the affected or failed nodes and links, and network geometry and size. In terms of recovery budget, the additional resources compared to small-scale disaster, will be approximately $50 \%$ and $90 \%$ more, after a medium and large-scale disaster scenario, respectively. However, in cases of higher penetration of CAV (e.g., 50\% or more), allowing such long recovery time will affect the operations of CAVs in the post-disaster transportation system. As a result, in cases of high CAV penetration, the recovery budget can increase significantly (due to assigning more recovery teams, more equipment, or both) to reduce the recovery time. However, for the road network used in this research, the recovery budget allowing half recovery time (compared to the time needed for smallscale recovery resources) was estimated to increase additional 10\% after the medium and largescale disaster scenarios, respectively. It represents that even a small change in recovery budget can gain significant improvement in recovery time consideration. 


\section{CHAPTER 6: CONCLUSIONS AND FUTURE RESEARCH DIRECTIONS}

\subsection{Conclusions}

The developed resilience model is capable of explaining the change in the resilience performance of transportation system in mixed-traffic environment. CAVs can assist disaster recovery activities by providing real time traffic information which can reduce the recovery time and effective utilization of limited resources. However, careful planning and deployment of CAV-supporting infrastructure is critical because repair and replacement of CAV infrastructure is expensive and requires specialized expertise.

This research formulated different resilience performance metrics such as, capacity, flow, travel time for a mixed-traffic environment. The disruption impact factor (DIF) introduced in this research is a new way to identify critical nodes in a network considering varying disaster severity, and associated impacts on the network. During the recovery phase, DIF factor can be used to identify nodes that need to be repaired first to gain maximum traffic operational efficiency improvement.

Furthermore, the analysis showed that system resilience (SR) followed a gradual decreasing trend in $\mathrm{P} 2$ in all the disaster scenarios compared to pre-disaster phase (P1) due to deteriorating roadway weather condition, higher following headway between vehicles, speed drop (reduction) and roadway capacity reduction. In the case of small-scale disaster scenario, the reduction of SR was $18 \%$ in $\mathrm{P} 2$ which mean $18 \%$ of the pre-disaster trips were disrupted due to the pre-disaster condition. Similarly, $29 \%$ and $41 \%$ trips were disrupted in medium and large-scale disaster scenario, respectively. The extent of recovery efforts is critical in recovering SR in P5. With similar recovery efforts in terms of budget and resources, maximum improvement in SR in P5 (compared to P4) was found for medium-scale disaster scenario (69\% resilience gain), while in small-scale and large-scale disaster scenario this improvement was $29 \%$ and $20 \%$ respectively. Though during P4, the SR in medium-scale and large-scale disaster scenarios were same (zero), resilience gain (repairing equal number of nodes) was disproportionate during P5 with same level of recovery budget for different severity level of disaster.

Some of the key limitations of this study include that this model does not capable to consider evacuation strategy in its present form. As evacuation is one of the most common and a widespread preparatory activity taken in almost all severe disaster scenarios to reduce the disaster 
consequences, this model may be modified in future to accommodate evacuation demand in its performance analysis. This can be done in Phase 2, as most of the evacuation demand will impact the performance of transportation network system during that phase. The five phase technique of classifying disaster impact can come in handy during quantifying resilience of the system in smaller timescale. In a nutshell, this model is a simplified framework of all the key properties of the transportation network system for mixed-traffic environment that influence the resilience of transportation system. This model, in its present form, is applicable to analyze a real world smallsized transportation network system and the impacts of small-medium scale disaster scenarios in which half portion of the network system may be unaffected. However, this model can further be modified to perform more robust analysis through involving more contributing parameters.

\subsection{Future Research Directions}

Several potential future extensions of this work are identified in this sub-section.

1. Evacuation strategy is a key component missing in this model. Further works can be done to incorporate the evacuation demand and the impact of evacuation in the performance of the system.

2. The model can be modified to perform more robust analysis considering large-sized network system and the impact of intense and severe disaster scenario.

3. As in real-world, most transportation networks operate in multimodal environment, formulating the resilience model considering a multimodal network will be very useful.

4. To include the post-disaster change in travel behavior, the proposed model needs to be revised to allow route choice modification based on the trip makers' new route preference.

5. Performance of post disaster transportation system depends on the post disaster regional activity pattern. Considering this new regional travel pattern in the resilience model will improve the accuracy of the model.

6. In this research, a hypothetical transportation network was used to evaluate the model performance. Follow up study using a real-world transportation network can provide more validity of the proposed model. 


\section{REFERENCES}

1. U.S. Billion-Dollar Weather and Climate Disasters. NOAA National Centers for Environmental Information (NCEI). https://www.ncdc.noaa.gov/billions/events/US/2017. Accessed June 10, 2018.

2. Faturechi, R., \& Miller-Hooks, E. (2014). Measuring the performance of transportation infrastructure systems in disasters: A comprehensive review. Journal of infrastructure systems, 21(1), 04014025.

3. Fok, E. (2013). An Introduction to Cybersecurity Issues in Modern Transportation Systems. ITE Journal, 3, 19.

4. Kelarestaghi, K. B., Heaslip, K., Fessmann, V., Khalilikhah, M., \& Fuentes, A. (2018). Intelligent Transportation System Security: Hacked Message Signs. Transp. Cybersecurity Priv., Unpublished.

5. Koscher, K., Czeskis, A., Roesner, F., Patel, S., Kohno, T., Checkoway, S., ... \& Savage, S. (2010, May). Experimental security analysis of a modern automobile. In Security and Privacy (SP), 2010 IEEE Symposium on (pp. 447-462). IEEE.

6. CASE and WMC, "Protection of Transportation Infrastructure from Cyber Attacks: A Primer, National Academies of Sciences, Engineering, and Medicine," NCHRP Document 221, 2015

7. Phase, I. (2008). Cooperative intersection collision avoidance system limited to stop sign and traffic signal violations.

8. Faturechi, R., \& Miller-Hooks, E. (2014). Travel time resilience of roadway networks under disaster. Transportation research part B: methodological, 70, 47-64.

9. Chen, L., \& Miller-Hooks, E. (2012). Resilience: an indicator of recovery capability in intermodal freight transport. Transportation Science, 46(1), 109-123.

10. Zhu, F., \& Ukkusuri, S. V. (2017). An optimal estimation approach for the calibration of the car-following behavior of connected vehicles in a mixed traffic environment. IEEE Transactions on Intelligent Transportation Systems, 18(2), 282-291.

11. Hannoun, G. J. (2017). Framework for better Routing Assistance for Road Users exposed to Flooding in a Connected Vehicle Environment (Doctoral dissertation, Virginia Tech). 
12. Zhao, J., Ma, W., Zhang, H. M., \& Yang, X. (2013). Two-step optimization model for dynamic lane assignment at isolated signalized intersections. Transportation Research Record, 2355(1), 39-48.

13. Zhao, J., Ma, W., Zhang, H., \& Yang, X. (2013). Increasing the capacity of signalized intersections with dynamic use of exit lanes for left-turn traffic. Transportation Research Record: Journal of the Transportation Research Board, (2355), 49-59.

14. Prevedouros, P. D., \& Chang, K. (2005). Potential effects of wet conditions on signalized intersection LOS. Journal of transportation engineering, 131(12), 898-903.

15. Rahman, M. M., Hasan, T., \& Nakamura, F. (2008). Development of professional driver adjustment factors for the capacity analysis of signalized intersections. Journal of Transportation Engineering, 134(12), 532-536.

16. Tang, T. Q., Li, P., \& Yang, X. B. (2013). An extended macro model for traffic flow with consideration of multi static bottlenecks. Physica A: Statistical Mechanics and Its Applications, 392(17), 3537-3545.

17. Darbha, S., \& Rajagopal, K. R. (1999). Intelligent cruise control systems and traffic flow stability. Transportation Research Part C: Emerging Technologies, 7(6), 329-352.

18. Yu, S., \& Shi, Z. (2015). The effects of vehicular gap changes with memory on traffic flow in cooperative adaptive cruise control strategy. Physica A: Statistical Mechanics And Its Applications, 428, 206-223.

19. Lindsey, R., Daniel, T., Gisches, E., \& Rapoport, A. (2014). Pre-trip information and routechoice decisions with stochastic travel conditions: Theory. Transportation Research Part B: Methodological, 67, 187-207.

20. Sun, H., Wu, J., Wang, W., \& Gao, Z. (2014). Reliability-based traffic network design with advanced traveler information systems. Information Sciences, 287, 121-130.

21. Karoonsoontawong, A., \& Lin, D. Y. (2011). Time-varying lane-based capacity reversibility for traffic management. Computer-Aided Civil and Infrastructure Engineering, 26(8), 632-646.

22. Omer, M., Mostashari, A., \& Nilchiani, R. (2013). Assessing resilience in a regional roadbased transportation network. International Journal of Industrial and Systems Engineering, 13(4), 389-408. 
23. Miller-Hooks, E., Zhang, X., \& Faturechi, R. (2012). Measuring and maximizing resilience of freight transportation networks. Computers \& Operations Research, 39(7), 1633-1643.

24. Donovan, B., \& Work, D. B. (2017). Empirically quantifying city-scale transportation system resilience to extreme events. Transportation Research Part C: Emerging Technologies, 79, 333-346.

25. Asadabadi, A., \& Miller-Hooks, E. (2017). Assessing strategies for protecting transportation infrastructure from an uncertain climate future. Transportation Research Part A: Policy and Practice, 105, 27-41.

26. Asadabadi, A., \& Miller-Hooks, E. (2017). Optimal transportation and shoreline infrastructure investment planning under a stochastic climate future. Transportation Research Part B: Methodological, 100, 156-174.

27. Asadabadi, A., \& Miller-Hooks, E. (2018). Co-opetition in enhancing global port network resiliency: A multi-leader, common-follower game theoretic approach. Transportation Research Part B: Methodological, 108, 281-298.

28. Kontou, E., Murray-Tuite, P., \& Wernstedt, K. (2017). Duration of commute travel changes in the aftermath of Hurricane Sandy using accelerated failure time modeling. Transportation Research Part A: Policy and Practice, 100, 170-181.

29. Freckleton, D., Heaslip, K., Louisell, W., \& Collura, J. (2012). Evaluation of resiliency of transportation networks after disasters. Transportation research record, 2284(1), 109-116.

30. Janić, M. (2015). Reprint of "Modelling the resilience, friability and costs of an air transport network affected by a large-scale disruptive event”. Transportation Research Part A: Policy and Practice, 81, 77-92.

31. Faturechi, R., Levenberg, E., \& Miller-Hooks, E. (2014). Evaluating and optimizing resilience of airport pavement networks. Computers \& Operations Research, 43, 335-348.

32. Fountoulakis, M., Bekiaris-Liberis, N., Roncoli, C., Papamichail, I., \& Papageorgiou, M. (2017). Highway traffic state estimation with mixed connected and conventional vehicles: Microscopic simulation-based testing. Transportation Research Part C: Emerging Technologies, 78, 13-33.

33. Wang, R., Li, Y., \& Work, D. B. (2017). Comparing traffic state estimators for mixed human and automated traffic flows. Transportation Research Part C: Emerging Technologies, 78, 95-110. 
34. Bahaaldin, K., Fries, R., Bhavsar, P., \& Das, P. (2017). A case study on the impacts of connected vehicle technology on no-notice evacuation clearance time. Journal of advanced transportation, 2017.

35. Future proofing infrastructure for connected and automated vehicles. Technical report. CATAPULT Transport Systems, 2017.

https://s3-eu-west-1.amazonaws.com/media.ts.catapult/wp-

content/uploads/2017/04/25115313/ATS40-Future-Proofing-Infrastructure-for-CAVs.pdf Accessed July 5, 2018.

36. Huang, Y., Fan, Y., \& Cheu, R. L. (2007). Optimal allocation of multiple emergency service resources for protection of critical transportation infrastructure. Transportation Research Record, 2022(1), 1-8.

37. Cappanera, P., \& Scaparra, M. P. (2011). Optimal allocation of protective resources in shortest-path networks. Transportation Science, 45(1), 64-80.

38. Rawls, C. G., \& Turnquist, M. A. (2010). Pre-positioning of emergency supplies for disaster response. Transportation research part B: Methodological, 44(4), 521-534.

39. Sen, A., Mazumder, A., Banerjee, J., Das, A., \& Compton, R. (2014). Identification of \$lcal K \$ Most Vulnerable Nodes in Multi-layered Network Using a New Model of Interdependency. arXiv preprint arXiv:1401.1783.

40. Hussain, O., Ghiasi, A., \& Li, X. (2016). Freeway Lane Management Approach in Mixed Traffic Environment with Connected Autonomous Vehicles.arXiv preprint arXiv:1609.02946.

41. Yang, X., \& Zhang, N. (2005). The marginal decrease of lane capacity with the number of lanes on highway. In Proceedings of the Eastern Asia Society for Transportation Studies (Vol. 5, pp. 739-749).

42. Chandra, S., \& Kumar, U. (2003). Effect of lane width on capacity under mixed traffic conditions in India. Journal of transportation engineering, 129(2), 155-160.

43. Kononov, J., Durso, C., Reeves, D., \& Allery, B. K. (2012). Relationship between traffic density, speed, and safety and its implications for setting variable speed limits on freeways. Transportation Research Record, 2280(1), 1-9.

44. Hosseini, S., Barker, K., \& Ramirez-Marquez, J. E. (2016). A review of definitions and measures of system resilience. Reliability Engineering \& System Safety, 145, 47-61. 
45. M. Hofmann, \& M. O'Mahony. The impact of adverse weather conditions on urban bus performance measures an analysis using ITS technology. In IEEE Conference on Intelligent Transport Systems, Vienna, 2005.

46. Lam, W. H., Shao, H., \& Sumalee, A. (2008). Modeling impacts of adverse weather conditions on a road network with uncertainties in demand and supply. Transportation research part B: methodological, 42(10), 890-910.

47. Hranac, R., E. Sterzin, D. Krechmer, H. A. Rakha, M. Farzaneh, and M. Arafeh. Empirical studies on traffic flow in inclement weather. Technical Report. USDOT FHA, 2006. https://ops.fhwa.dot.gov/publications/weatherempirical/weatherempirical.pdf Accessed by July 7, 2018.

48. Mínguez, R., Sánchez-Cambronero, S., Castillo, E., \& Jiménez, P. (2010). Optimal traffic plate scanning location for OD trip matrix and route estimation in road networks. Transportation Research Part B: Methodological, 44(2), 282-298. 